\title{
Transferencia y permanencia del entrenamiento dual $n$-back en la inteligencia fluida y en la memoria de trabajo verbal
}

\author{
Marcelo Andrés Panza Lombardo ${ }^{1}$
}

\begin{abstract}
Artículo
Material original autorizado para la publicación en la revista Psicodebate. Facultad de Ciencias Sociales. Universidad de Palermo.

Recibido 09-11-2015 | Aceptado 12-02-2016
\end{abstract}

\section{Resumen}

Existen polémicas con respecto a la posibilidad de aumentar los niveles de inteligencia fluida y de memoria de trabajo por medio de entrenamiento en tareas complejas de memoria de trabajo. La finalidad de los tres experimentos realizados fue constatar efectos de transferencia y permanencia en la memoria de trabajo verbal y en la inteligencia fluida, a partir del entrenamiento en el programa dual n-back. En el primer estudio se evaluó la diferencia extra-grupo en el entrenamiento dual n-back luego de 25 sesiones, comparando los resultados del grupo experimental con los del grupo control activo. En el segundo estudio se compararon los efectos del entrenamiento en el programa dual n-back luego de 100 sesiones. El tercer experimento evaluó el efecto de 25, 50, 75 y 100 sesiones de entrenamiento. El último estudio consistió en medir la permanencia de los efectos del entrenamiento a los $1,2,4$, y 6 meses de terminado el mismo. Los resultados sugieren la posibilidad de aumentar medidas de inteligencia fluida y de memoria de trabajo por medio del entrenamiento dual $n$-back, y a su vez la permanencia de estos efectos.

Palabras Clave: inteligencia fluida, memoria de trabajo verbal, entrenamiento dual-n back, transferencia, permanencia.

1 Universidad Privada del Este (Presidente Franco, Paraguay), Sensorium, departamento de formación (Ciudad del Este, Paraguay); marcelopanza@hotmail.com 


\title{
Transference and retention of the dual $\mathbf{n}$-back training in fluid intelligence and verbal working memory
}

\begin{abstract}
There are controversies regarding the possibility of increasing the levels of fluid intelligence and working memory through training in working memory tasks. The purpose of the three experiments was to evaluate effects of the transference and retention in verbal working memory and fluid intelligence, from training in the dual n-back program. In the first study the extra-group difference was evaluated in the dual n-back training after 25 sessions, comparing the results of the experimental group with those of the active control group. In the second study the effects of training in the dual n-back program were evaluated after 100 sessions. The third experiment evaluated the effect of 25, 50, 75 and 100 training sessions. The latest study measured the permanence of the effects of training, at 1,2, 4, and 6 months after the completion of training. The results suggest the possibility of increasing measures of fluid intelligence and working memory using the dual n-back training, and in turn the permanence of these effects.
\end{abstract}

Keywords: fluid intelligence, verbal working memory, dual n-back training, transference, retention. 
La inteligencia fluida y la memoria de trabajo son dos mecanismos que presentan múltiples coincidencias. En primer lugar, ambos mecanismos poseen elevado poder predictivo en comportamientos de vital importancia para el ser humano. La inteligencia fluida es considerada como el principal predictor del rendimiento laboral (Schmidt, 2009; Schmidt \& Hunter, 1998, 2004) y del rendimiento académico (Deary, Strand, Smith \& Fernandes, 2007; Watkins, Lei \& Canivez, 2007), mientras que la memoria de trabajo también es considerada por las investigaciones de Alloway (2009) y, Alloway y Alloway (2010) como el principal mecanismo de predicción del rendimiento académico, y diversos autores reportan su rol central en el éxito académico (Loosli, Buschkuehl, Perrig \& Jaeggi, 2012; Rojas-Barahona, Förster, Moreno-Ríos \& McClelland, 2015; Titz \& Karbach, 2014; Wiley \& Jaroz, 2012; Yuan, Steedle, Shavelson, Alonzo, \& Oppezzo, 2006).

A su vez, la inteligencia fluida y la memoria de trabajo son dos mecanismos fuertemente asociados en aspectos psicométricos (Ackerman, Beier \& Boyle, 2005; Engel de Abreu, Conway \& Gathercole, 2010; Kane, Conway \& Hambrick, 2005) -a pesar de Gignac y Watkins (2015)-, neurológicos (Barbey, Colom, Paul, \& Grafman, 2014; Burgess, Gray, Conway, \& Braver, 2011), mecanísmicos (Colom et al., 2015; Engle, Tuholski, Laughlin, \& Conway, 1999; Unsworth, 2010), presentando niveles similares de heredabilidad (Darst et al., 2015; Davies et al., 2011) y de desarrollo (Horn, 1972; Kaufman, Kaufman-Packer, Mc Lean, \& Reinolds, 1991; Salthouse, \& Babcok, 1991; van den Noort, Haverkort, Bosch, Hugdahl, 2006).

A pesar de la fuerte determinación genética de ambos mecanismos, Jaeggi, Buschkuehl, Jonides y Perrig (2008), mediante 8, 12, 17 y 19 sesiones de entrenamiento de la memoria de trabajo con el programa dual n-back, lograron aumentar los niveles de inteligencia fluida y los niveles de memoria de trabajo verbal en el Test de Amplitud de Dígitos. En una investigación posterior, Jaeggi et al. (2010), lograron elevar los niveles de inteligencia fluida utilizando el programa dual n-back, y también un programa simplificado single $n$-back, por medio de 20 sesiones de entrenamiento. Sin embargo, en esta investigación no se obtuvieron aumentos en los niveles de memoria de trabajo.

Los estudios antecedentes sugerían la posibilidad de modificar tanto la inteligencia fluida como la memoria de trabajo por medio del entrenamiento dual n-back. Sin embargo, existían fuertes reparos metodológicos a tener en cuenta para interpretar los resultados. Mody (2009) sostuvo que los efectos podían deberse a la falta de validez de los instrumentos, puesto que los autores utilizaron versiones acortadas en el tiempo del Test de Matrices Progresivas de Raven y del BOMAT, restándole validez a las medidas de inteligencia fluida. Por su parte, Shipstead, Redick, \& Engle (2010) argumentaron que los resultados podían deberse al efecto Hawthorne y/o a la respuesta a la demanda, ya que no se utilizaron grupos de control activos. 
Teniendo en cuenta sólo la segunda de las críticas, los autores realizaron una tercera investigación, con grupo de control activo y una adaptación del programa single $n$-back, utilizando como participantes a niños. Sólo se obtuvieron resultados en la parte del grupo experimental que logró los mayores rendimientos en la tarea experimental de entrenamiento. Esos resultados se mantuvieron luego de un seguimiento efectuado a los 3 meses. Sin embargo, nuevamente utilizaron versiones acortadas de los instrumentos (Jaeggi, Buschkuehl, Jonides, \& Shah, 2011).

Posteriormente se presentaron 18 investigaciones que abordaron la temática con resultados dispares (Dougherty, Hamovitz, \& Tidwell, 2015). El meta-análisis de Melby-Lervåg, \& Hulme (2013), enfocado en el efecto del entrenamiento de la memoria de trabajo en diversas habilidades cognitivas, reportó efectos específicos de corto plazo y no generalizables. Contrariamente, el meta-análisis de Au et al. (2014), al abordar el efecto del entrenamiento de la memoria de trabajo en la inteligencia fluida, informa sobre un efecto pequeño pero significativo. Al respecto, Melby-Lervåg y Hulme (2015) contestaron que dicho efecto se debía a la inclusión de estudios sin grupo control, con otros déficits metodológicos, y a la falta de consideración de las diferencias de línea base en el cálculo del tamaño del efecto. Au, Buschkuehl, Duncan, \& Jaeggi, (2015) respondieron mostrando que en las comparaciones entre los estudios con grupo control y sin grupo control no existían diferencias significativas de efecto. Mientras que dos meta-análisis, con respecto al efecto del entrenamiento en la memoria de trabajo, concluyen en la existencia de transferencia cercana y lejana a la memoria de trabajo de tamaño pequeño a medio (Karbach, \& Verhaeghen, 2014; Schwaighofer, Fischer, \& Bühner, 2015). Utilizando estadística bayesiana (Dougherty et al., 2015), sostienen que existen diferencias claras entre los estudios que utilizan grupo control y aquellos que no, por lo que hace necesaria la utilización de los mismos, y deja en duda que realmente existan efectos de transferencia sobre la inteligencia fluida.

Las investigaciones referidas no permitían afirmar que el entrenamiento en el programa dual n-back aumente la inteligencia fluida y la memoria de trabajo. Era necesaria la medición con instrumentos utilizados de la misma manera en que habían sido validados $\mathrm{y}$, a su vez, se hacía necesaria la utilización de grupo control activo. Esto se hacía mandatorio, dado que, cuando se utilizó el grupo control activo con niños, los autores no pudieran replicar sus resultados en la totalidad del grupo experimental (Jaeggi et al., 2011), y por los resultados reportados por Dougherty et al. (2015).

Se hacía necesario también conocer los efectos del entrenamiento mediante el programa dual n-back por más de 20 sesiones. También existían interrogantes con respecto a la permanencia de los efectos del entrenamiento en adultos. Si bien los resultados iniciales de Jaeggi et al. (2008) sugerían que a más sesiones se logran 
mayores resultados, esto no se ha reportado en Jaeggi et al. (2011). Otro aspecto que no se había estudiado era el efecto del entrenamiento con 2 sesiones diarias. Por último, no estaba claro el efecto que producía el entrenamiento dual n-back en la memoria de trabajo. La presente investigación tuvo como objetivo abarcar los interrogantes mencionados.

Teniendo en cuenta los datos antecedentes, se plantearon las siguientes hipótesis:

H1. El entrenamiento dual n-back producirá aumentos en la inteligencia fluida luego de 25 sesiones de entrenamiento.

H2. El entrenamiento dual n-back producirá aumentos en la memoria de trabajo verbal luego de 25 sesiones de entrenamiento.

H3. Se registrará un aumento de la inteligencia fluida mayor en el entrenamiento de 100 sesiones en comparación con el de 75 sesiones, en el entrenamiento de 75 sesiones en comparación con el de 50 sesiones, y en el entrenamiento de 50 sesiones en comparación con el de 25 sesiones, y en el entrenamiento de 25 sesiones.

H4. Se registrará un aumento de la memoria de trabajo verbal mayor en el entrenamiento de 100 sesiones en comparación con el de 75 sesiones, en el entrenamiento de 75 sesiones en comparación con el de 50 sesiones, y en el entrenamiento de 50 sesiones en comparación con el de 25 sesiones, y en el entrenamiento de 25 sesiones en comparación con la ausencia de entrenamiento en el grupo experimental.

H5. El grupo experimental presentará niveles mayores de inteligencia fluida que el grupo control, luego de 1, 2, 4, y 6 meses de terminado el entrenamiento.

H6. El grupo experimental presentará niveles mayores de memoria de trabajo verbal que el grupo control, luego de 1, 2, 4, y 6 meses de terminado el entrenamiento.

\section{Estudio 1}

En el experimento 1 se pusieron a prueba las hipótesis 1 y 2, las cuales sostenían que el programa dual $n$-back produciría aumentos en la inteligencia fluida y en la memoria de trabajo verbal. Teniendo en cuenta las críticas metodológicas, se utilizó un grupo control activo (Dougherty et al., 2015; Melby-Lervåg \& Hulme, 2015; Shipstead et al., 2010).

\section{Método}

\section{Participantes}

Teniendo en cuenta el tamaño muestral sugerido para las pruebas estadísticas de diferencia de grupo (Cowles, 1974), y los antecedentes de abandono con el programa 
(Jaeggi et al., 2008), se seleccionaron 80 participantes, estudiantes universitarios de una institución de Ciudad del Este, a partir de los siguientes criterios de inclusión: a) Cercanía al laboratorio; b) Disponibilidad de tiempo; c) Nivel básico de inglés; d) Nivel de sinceridad (Eysenck \& Eysenck, 1985/1997) medio a elevado; e) Edad de 18 a 35 años; f) Ausencia de déficits visuales y auditivos; g) Niveles menores a 30 puntos para el Test de Raven, 30 puntos para el Test G, 13 puntos para el Test Amplitud de Dígitos y 40 puntos para la Prueba de Amplitud Lectora. De esos participantes quedaron 75 luego de la primeras 25 sesiones, por lo que se analizarán los datos de los participantes remanentes, ya que con estos se efectuó la investigación. 45 participantes (60\%) fueron de sexo femenino y 30 (40\%) de sexo masculino. Las edades de los participantes variaron entre los 18 y los 33 años, siendo la media de edad de $22.12 \pm$ 2.504 años. El grupo experimental quedó conformado por 35 participantes, mientras que el grupo control, por 40 participantes. No existieron diferencias estadísticamente significativas en cuanto a edad ni sexo entre los grupos. A todos los participantes se les otorgó una remuneración una vez completados los tres estudios.

\section{Materiales}

\section{Medición de la inteligencia fluid}

Test de Matrices Progresivas de Raven, Escala Avanzada (Raven, Court \& Raven, 1976/1999). Instrumento de completamiento de matrices progresivas a partir de sus relaciones lógicas. Se tomó el instrumento con sus limitaciones de tiempo establecidas, y se corrigió a partir de su puntaje bruto. En numerosas investigaciones, el instrumento es considerado como la mejor medida de la inteligencia fluida (Basak, Boot, Voss, \& Kramer, 2008; Burgess et al., 2011; Gray, Chabris, \& Braver, 2003; Jaeggi et al., 2008; Jaeggi et al., 2010; Kane \& Engle, 2002; Klingberg, Forssberg \& Westerberg, 2002).

Test Libre de Influencias Culturales, G, escala 3 de Cattell (Cattell \& Cattell, 1973/2001). Instrumento de matrices que evalúa la inteligencia fluida a partir de identificación y de ejecución de relaciones lógicas. Se tomó el instrumento con sus limitaciones de tiempo establecidas, y se corrigió a partir de su puntaje bruto. El instrumento fue seleccionado debido a que ha sido construido por uno de los creadores del concepto de inteligencia fluida con la finalidad de medir tal proceso (Carroll, 1993; Cattell, 1987), y ha sido utilizado en estudios relacionados con la temática a investigar (Borella, Carretti, Riboldi \& De Beni, 2010; Tranter \& Koutstaal, 2008).

\section{Medición de la memoria de trabajo verbal}

Sub-escala Amplitud de Dígitos del Test WAIS (Weschler, 1984). Instrumento de amplitud de dígitos, que posee dos tipos de tareas: a) dígito hacia adelante, la 
cual consiste en repetir los números dictados por el evaluador en el orden que han sido escuchados; b) dígito hacia atrás, consistente en repetir los números en orden inverso al que han sido escuchados. Se lo considera como una medida fiable de memoria de trabajo verbal (Ackerman et al., 2002; Borella et al., 2010; Oberauer, Suß, Schulze, Wilhelm \& Wittmann, 2000; Rosen, \& Engle, 1997; Yuan, Steedle, Shavelson, Alonzo, \& Oppezzo, 2006).

Prueba de Amplitud Lectora PAL, adaptación española del Reading Span Test de Daneman y Carpenter (Elosúa, Gutierrez, García Madruga, Luque \& Gárate, 1996). Consta de seis frases de entrenamiento, y 60 frases experimentales, repartidas en 3 series para cada nivel, teniendo 5 niveles, que van de 2 palabras a recordar a 6 . Se adoptó el criterio descriptivo propuesto por los autores de la adaptación española (Elosúa et al., 1996). Si bien Elosúa et al. (1996) consideraron que el instrumento mide la memoria de trabajo general, Barreyro, Burin y Duarte (2009), al realizar la adaptación y validación del instrumento a la Argentina, obtuvieron resultados que permiten sostener que la tarea es válida para medir la memoria de trabajo verbal, por lo cual se la considerará como una medida de la misma.

\section{Validación de los instrumentos}

Se evaluó la confiabilidad y validez de los cuatro instrumentos. El proceso se realizó a partir de una muestra de 130 alumnos universitarios de una institución de Ciudad del Este, con una media de edad de $23.08 \pm 3.7$, siendo el mínimo de edad de 18 y el máximo de 37 . La cantidad de participantes mujeres fue de 83 y la cantidad de participantes hombres fue de 47. Se obtuvieron las siguientes medidas de confiabilidad, validez de constructo concurrente y validez de criterio.

Tabla 1.

Confiabilidad: Coeficientes $\alpha$ de Cronbach para los cuatro instrumentos.

\begin{tabular}{ccccc}
\hline & Raven & Test $\mathbf{g}$ & Dígito & P.A.Lectora \\
\cline { 2 - 5 }$\alpha$ & .829 & .798 & .803 & .779 \\
\hline
\end{tabular}

Tabla 2.

Validez de constructo concurrente: Correlaciones entre los cuatro instrumentos.

\begin{tabular}{lcccc}
\hline & Raven & G & Dígito & PAL \\
\cline { 2 - 5 } Raven & ---- & & & \\
G & .848 & ---- & & \\
Dígito & .715 & .833 & $-\cdots$ & \\
PAL & .698 & .874 & .759 & --- \\
\hline
\end{tabular}


Para la validez de criterio se correlacionaron los puntajes obtenidos en los test con el promedio de las calificaciones académicas del último año de la secundaria.

Tabla 3.

Validez de criterio: Correlaciones entre los instrumentos y las calificaciones académicas.

\begin{tabular}{lcc}
\hline & $\begin{array}{c}\text { Promedio de } \\
\text { calificaciones }\end{array}$ & $\boldsymbol{P}$ \\
\hline Raven & .743 & .000 \\
$\mathrm{G}$ & .503 & .000 \\
Dígito & .299 & .01 \\
Amplitud Lectora & .401 & .000 \\
\hline
\end{tabular}

\section{Programa de entrenamiento dual n-back}

Software diseñado para entrenar la memoria de trabajo a partir del paradigma dual n-back (Buschkuehl, Jaeggi, Kobel \& Perrig, 2007). Es el mismo software que el utilizado por Jaeggi et al, $(2008,2010)$. El entrenamiento consiste en lo siguiente: cuadrados en ocho posiciones posibles son presentados de manera secuencial en la pantalla de la computadora a una razón de tres segundos, con una duración del estímulo de 500 milisegundos, y una distancia de un estímulo a otro de 2500 milisegundos. Simultáneamente a la presentación de los cuadrados, una de ocho consonantes en inglés es presentada auditivamente en los altoparlantes o auriculares. El participante debe presionar una tecla (la L o la S) cuando alguno de los cuadrados o consonantes presentados coincida con un cuadrado o consonante anterior en una posición $n$ de la secuencia. El valor de $n$ es el mismo para los dos estímulos, comenzando con $n=1$, es decir, se debe apretar la tecla si el mismo estímulo se repite de manera inmediata. Los participantes responden de manera manual presionando, en un teclado estándar, la letra "S" para la coincidencia de la visualización de cuadrados, y la letra "L" para la coincidencia de la audición de letras. No son requeridas respuestas para las presentaciones que no coincidan con las anteriores. Por ejemplo, si en la primera presentación surge un cuadrado en un lugar y se escucha la consonante "Be", y en la segunda presentación surge un cuadrado en la misma posición y se repite nuevamente la consonante "Be", se debería tocar tanto la tecla S (el cuadrado anterior coincide con el actual), como la tecla L (la consonante anterior coincide con la actual). El nivel de dificultad varía a partir del rendimiento del participante. Luego de cada bloque, el rendimiento del participante es informado si el participante cometió menos de tres errores en la modalidad visual (cuadrado); y en la modalidad auditiva (letra), el nivel $n$ se incrementa en uno, es decir, ahora deberá apretar el botón si los estímulos tienen una separación de un 
estímulo de por medio, si ha cometido más de cinco errores en alguna modalidad, el nivel $n$ disminuye en uno. En los demás casos, el nivel de $\mathrm{n}$ es conservado. Las sesiones constan de 20 bloques de un minuto de duración, más un bloque por cada nivel alcanzado. La duración total de la sesión es de aproximadamente 25 minutos.

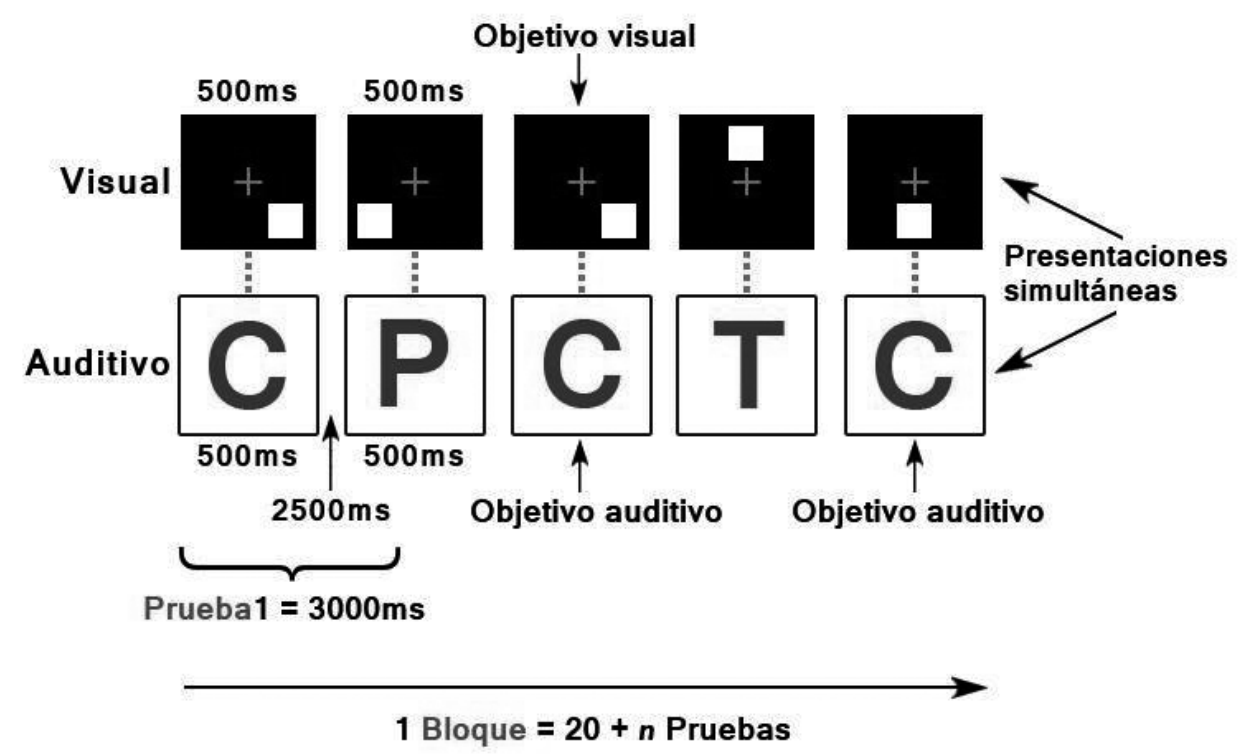

Figura 1. Ejemplo ilustrativo del programa Dual n-back.

\section{Entrenamiento en aritmética}

Es un entrenamiento en velocidad y precisión aritmética mediante material impreso. Se realizó una adaptación, inspirada en el libro Matemáticas Rápidas (Julius, 1998), de estrategias para aumentar la velocidad aritmética, y de 1362 ejercicios aritméticos de práctica. El entrenamiento consistió en el autoaprendizaje de estrategias para aumentar la velocidad y precisión aritmética, la realización de ejercicios para aplicar tales estrategias, y la autocorrección de dichos ejercicios.

\section{Diseño}

Diseño experimental, pre-prueba, post prueba, con grupo control activo.

\section{Procedimientos}

Los 80 participantes fueron asignados aleatoriamente a dos grupos: el grupo experimental y el grupo control. La aleatorización se efectuó a través del sorteo en un bolillero, y se le asignó a cada participante un número. Para confirmar la igualdad de los grupos, posteriormente se procedió al análisis estadístico de las características de los mismos. 
El grupo control constó inicialmente de 40 participantes, pero se sufrió una mortandad de la muestra de 5 participantes antes de terminar las 25 sesiones iniciales de entrenamiento. Por lo tanto, el grupo experimental estuvo integrado por 35 participantes (46.7\%), y el grupo control por 40 participantes $(53.3 \%)$.

Como pre-test se utilizaron las medidas tomadas previamente a los participantes, integradas por los siguientes instrumentos, en el orden aquí detallado: a) Test de Raven, Escala Avanzada; b) Test Amplitud de Dígitos; c) Test G de Cattell; d) Prueba de Amplitud Lectora.

El grupo experimental se entrenó en 2 sesiones diarias por 18 días ( 25 sesiones) en el programa dual n-back, mientras que el grupo control activo se entrenó en la resolución de operaciones aritméticas, por el mismo tiempo y frecuencia que el grupo experimental. El grupo experimental asistió de lunes a viernes en cuatro turnos disponibles a la mañana, lo cual a veces no se cumplió, ya que estos participantes disponían de tres horarios opcionales de tarde para asistir, mientras que el grupo control activo asistió de tarde, en cuatro turnos. Luego de los 18 encuentros, los participantes del grupo control activo llegaron hasta la página 301, en promedio.

Durante las 25 sesiones (18 encuentros), 5 integrantes del grupo control dejaron de asistir, aduciendo problemas con los horarios. El experimento culminó en el encuentro número 19, en el cual se re-evaluaron los niveles de inteligencia fluida y de memoria de trabajo verbal, que fueron medidos por cuatro instrumentos en el siguiente orden: a) Test de Raven, Escala Avanzada; b) Test Amplitud de Dígitos; c) Test G; d) Prueba de Amplitud Lectora.

\section{Análisis de datos}

Los datos fueron analizados con el software SPSS 19. Se optó por un nivel de significación de $p=.05$. En los datos que cumplieron con los presupuestos de la estadística paramétrica (distribución normal y homocedasticidad) se utilizó la prueba de diferencia de grupo $t$ de Student para muestras relacionadas, en las comparaciones intra-grupo, y $t$ de Student para muestras independientes en las comparaciones extra-grupo. Para los datos que no cumplieron con los presupuestos de la estadística paramétrica, se utilizaron pruebas no paramétricas como la $\mathrm{W}$ de Wilcoxon en las comparaciones intra-grupo, y la U de Mann Whitney, para comparaciones extra-grupo.

\section{Resultados}

Los valores medios de los cuatro test realizados antes del comienzo del entrenamiento para ambos grupos (pre-test) se exponen en la siguiente tabla: 
Tabla 4.

Media de cada instrumento en el grupo experimental y control en el pre-test.

\begin{tabular}{lcccc}
\hline & & $\boldsymbol{M}$ & $\boldsymbol{D E}$ & $\boldsymbol{p}$ \\
\hline Raven Preprueba & Grupo Experimental & 20.77 & 3.32 & .859 \\
& Grupo Control & 20.63 & 3.71 & \\
& & & & \\
Test g Preprueba & Grupo Experimental & 16.94 & 2.67 & .947 \\
& Grupo Control & 16.90 & 2.89 & \\
Dígito Preprueba & Grupo Experimental & 10.06 & 1.64 & .685 \\
& Grupo Control & 10.23 & 1.88 & \\
PAL Preprueba & Grupo Experimental & 18.83 & 4.85 & $.986^{\mathrm{a}}$ \\
& Grupo Control & 18.85 & 5.53 & \\
\hline
\end{tabular}

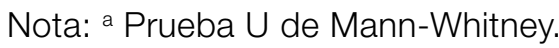

No se observaron diferencias estadísticamente significativas, por lo que se estableció la equivalencia inicial en cuanto a las variables dependientes.

Tabla 5.

Media de cada instrumento en el grupo experimental y control post-test.

\begin{tabular}{lccc}
\hline & & $\boldsymbol{M}$ & $\boldsymbol{D E}$ \\
\hline \multirow{2}{*}{ Raven Postprueba } & Grupo Experimental & 23.31 & 3.563 \\
& Grupo Control & 20.65 & 3.840 \\
Test g Postprueba & Grupo Experimental & 21.11 & 3.179 \\
& Grupo Control & 17.23 & 2.957 \\
Dígito Postprueba & Grupo Experimental & 11.31 & 1.491 \\
& Grupo Control & 10.20 & 2.078 \\
PAL Postprueba & Grupo Experimental & 23.97 & 5.997 \\
& Grupo Control & 18.30 & 5.557 \\
\hline
\end{tabular}


Tabla 6.

Diferencias intragrupo pre-test - post-test y extra-grupo post-test.

\begin{tabular}{lcccccccccc}
\hline Medida & \multicolumn{2}{c}{$\begin{array}{c}\text { Grupo Experimental } \\
\text { Pre y Post 25 }\end{array}$} & \multicolumn{4}{c}{$\begin{array}{c}\text { Grupo Control } \\
\text { Pre y Post } 25\end{array}$} & \multicolumn{2}{c}{$\begin{array}{c}\text { Diferencia entre } \\
\text { grupos Post-test }\end{array}$} & $\begin{array}{c}\text { Tamaño del } \\
\text { efecto* }^{*}\end{array}$ \\
\hline Raven & $\boldsymbol{t}$ & $\boldsymbol{g l}$ & $\boldsymbol{p}$ & $\boldsymbol{t}$ & $\boldsymbol{g l}$ & $\boldsymbol{p}$ & $\boldsymbol{t}$ & $\boldsymbol{g l}$ & $\boldsymbol{p}$ & $\boldsymbol{d}$ de Cohen \\
Test g & 10.90 & 34 & .000 & .085 & 39 & .933 & 3.10 & 73 & .003 & .70 \\
Dígito & 20.15 & 34 & .000 & 1.39 & 39 & .171 & 5.48 & 73 & .000 & 1.25 \\
\hline
\end{tabular}

Nota: *Sobre diferencia extra-grupo.

Se obtuvieron diferencias estadísticamente significativas, tanto en la comparación intra-grupo en el grupo experimental, como en la comparación entre el grupo experimental y el grupo control en el post-test, con tamaños del efecto medios para la inteligencia fluida medida por el test de Raven y para la memoria de trabajo medida por el test dígito, y elevados para la inteligencia fluida medida por el test $\mathrm{G}$. Con respecto a la Prueba de Amplitud Lectora, utilizando la prueba Wilcoxon para muestras relacionadas, se determinaron diferencias estadísticamente significativas intra-grupo entre el pre-test y post-test del grupo experimental $(p<.0001)$; mientras que no existieron diferencias estadísticamente significativas intra-grupo entre el pretest y el post-test del grupo control $(p=.135)$.

Se compararon los resultados en el post-test de la Prueba de Amplitud Lectora en el grupo experimental y el grupo control, por medio de la prueba U de MannWhitney para muestras independientes.

Tabla 7.

Diferencias extra-grupo para la Prueba de Amplitud Lectora.

\begin{tabular}{ccccc}
\hline $\boldsymbol{U}$ & $\boldsymbol{W}$ & $\boldsymbol{Z}$ & $\boldsymbol{P}$ & $\boldsymbol{r}^{*}$ \\
\hline 328 & 1148 & -4.006 & .000 & .46 \\
\hline
\end{tabular}

Nota: * Tamaño del efecto según la fórmula de Rosenthal (1991).

Como puede observarse, se obtuvieron diferencias estadísticamente significativas en la Prueba de Amplitud Lectora, con un tamaño del efecto medio (Rosenthal, 1991).

Por todo lo antecedente, los datos apoyan las hipótesis 1 y 2: el entrenamiento dual $n$-back produjo aumentos en los niveles de inteligencia fluida y de memoria de trabajo verbal luego de 25 sesiones de entrenamiento. 


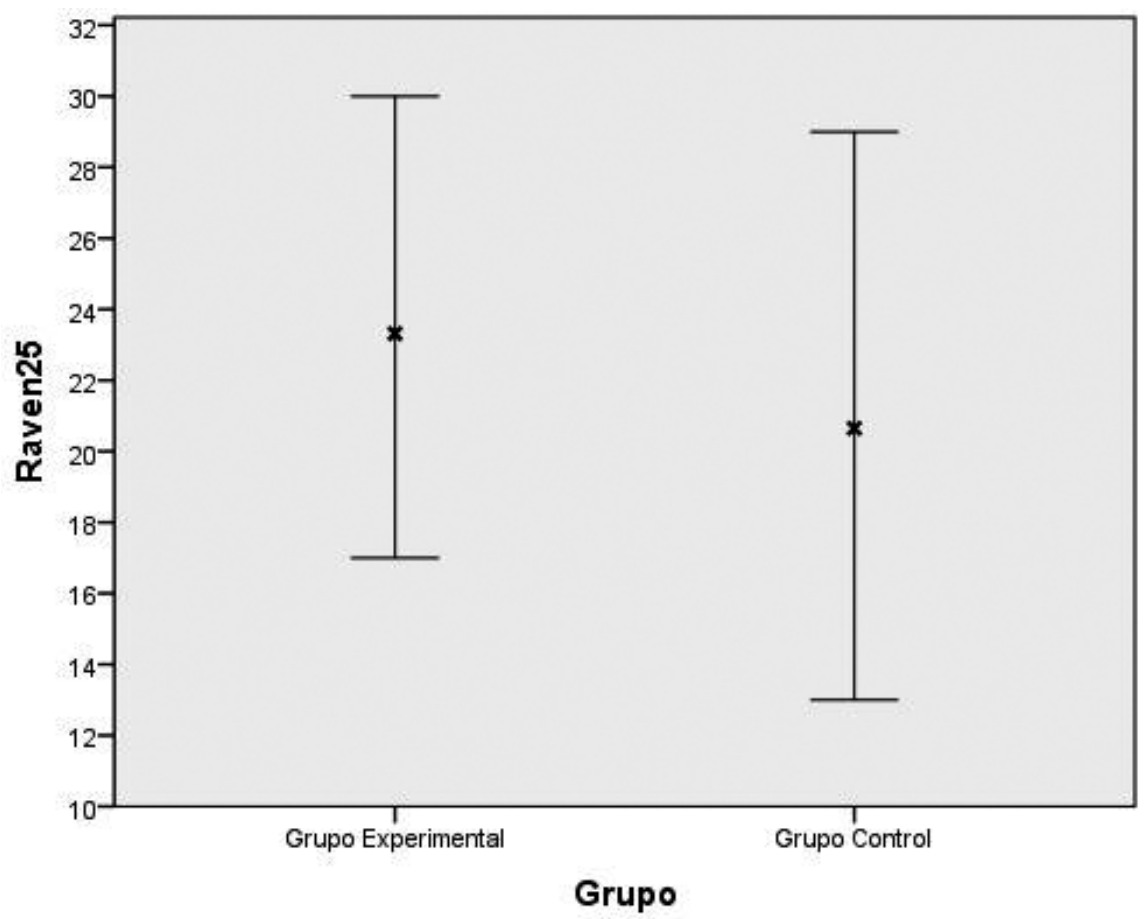

Figura 2. Distribución puntajes del grupo experimental y del grupo control en el test de Raven, post-test.

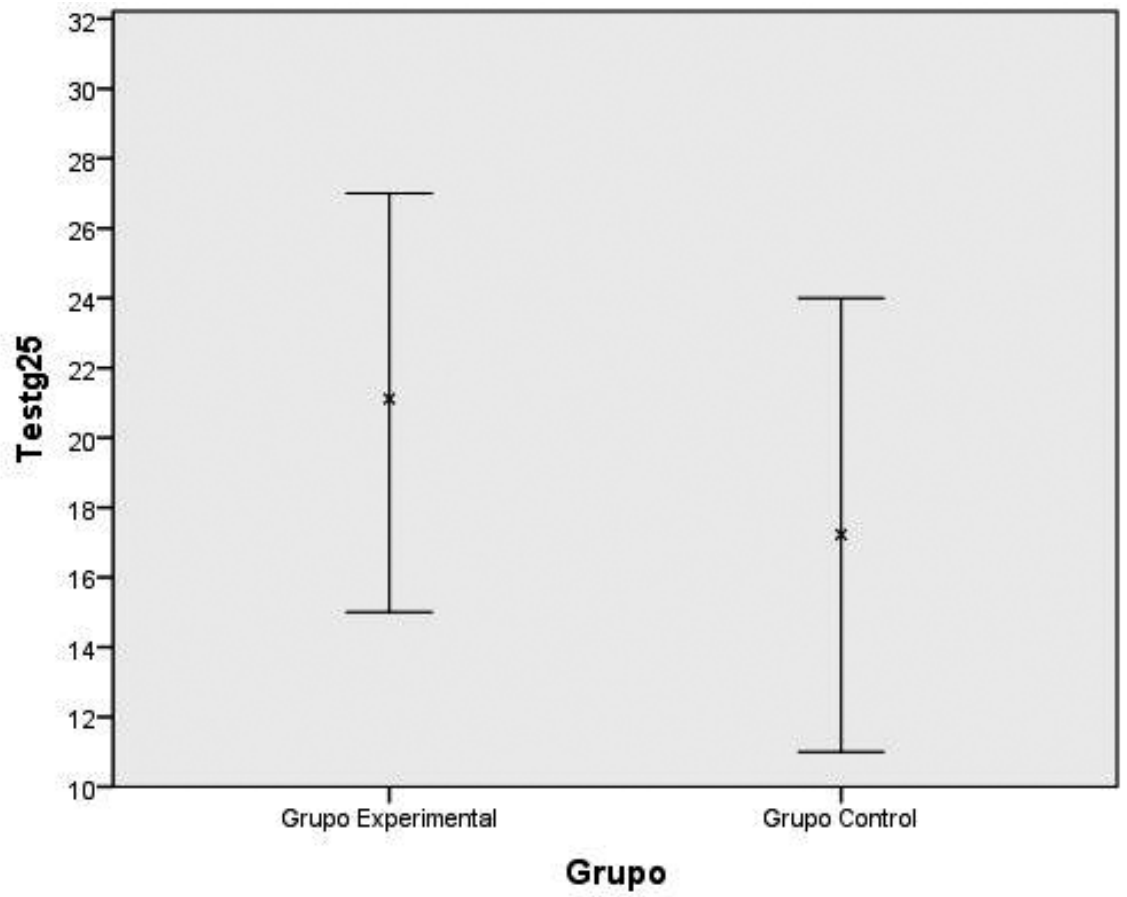

Figura 3. Distribución de los puntajes del grupo experimental y del grupo control en el test G de Cattell, post-test. 


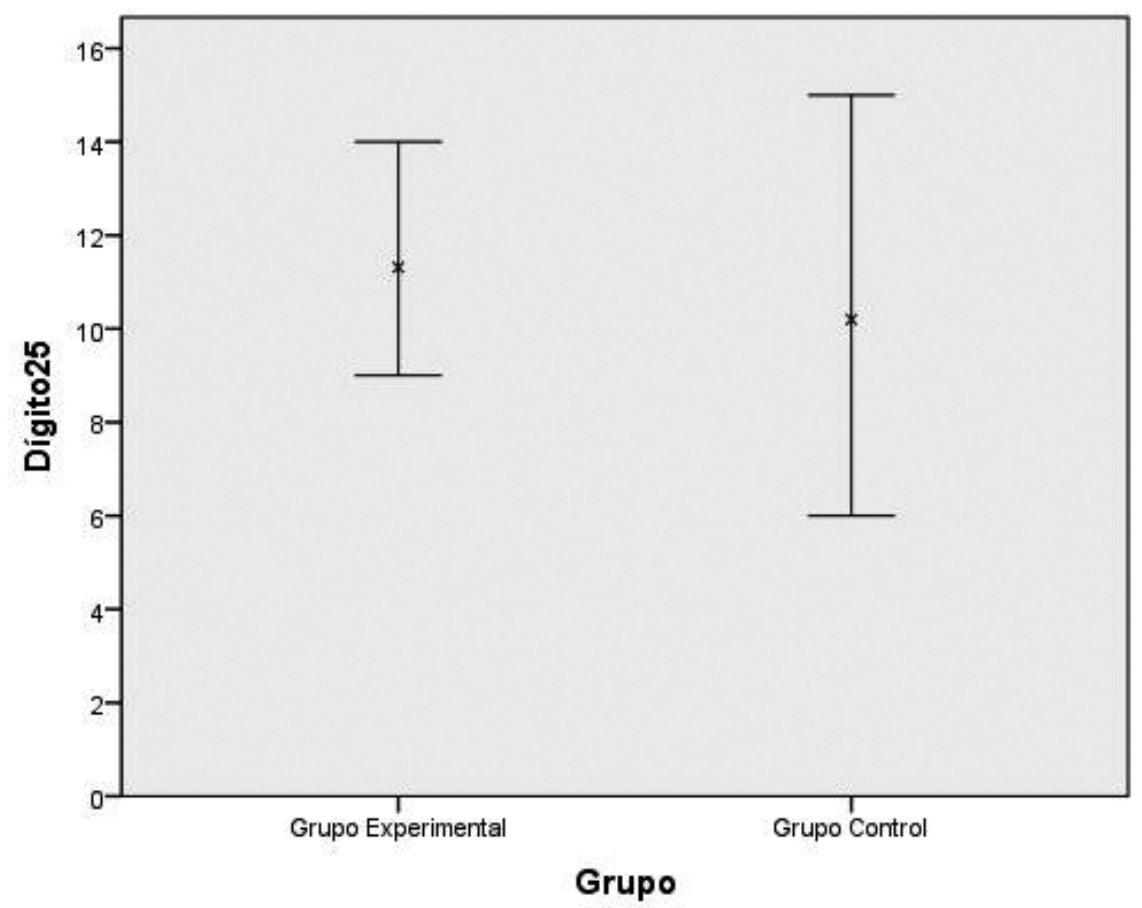

Figura 4. Distribución puntajes grupo experimental y grupo control en el test Dígito, post-test.

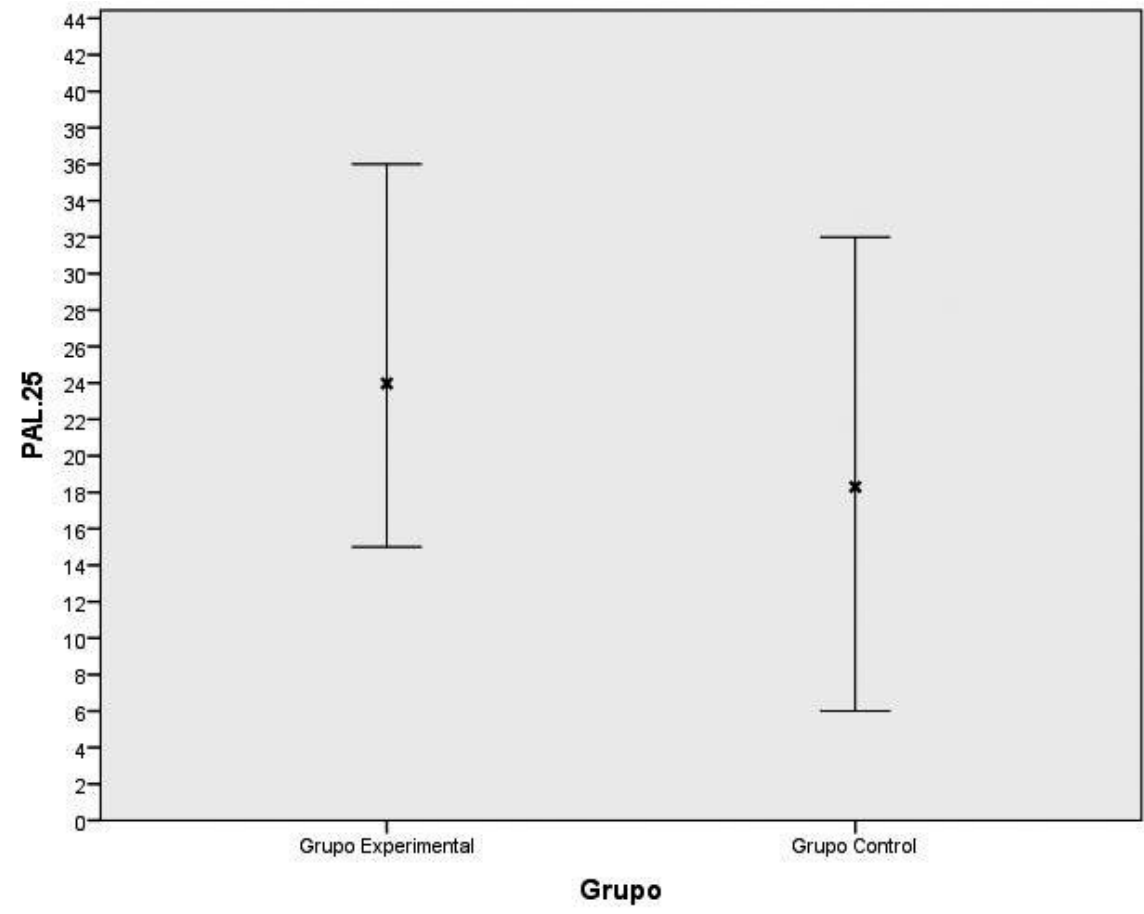

Figura 5. Distribución de los puntajes de la Prueba de Amplitud Lectora para el grupo experimental y el grupo control, post-test. 


\section{Estudio 2}

La finalidad del experimento 2 fue la de poner a prueba las hipótesis 3 y 4 , que afirmaban un mayor aumento de los niveles de inteligencia fluida y de memoria de trabajo verbal en el grupo experimental, cuanto más bloques de sesiones de entrenamiento se realizaran.

\section{Participantes}

Se emplearon los mismos participantes del experimento 1 y $2: 35$ participantes en el grupo experimental, y 40 participantes en el grupo control.

\section{Instrumentos}

Se emplearon los mismos instrumentos que en el experimento 1 y 2 .

\section{Diseño}

Diseño experimental, de series cronológicas con repetición de estímulo y grupo de control activo en las primeras 25 sesiones y grupo de control pasivo en las restantes 75 sesiones.

\section{Procedimientos}

Como pre-test se utilizaron los datos del experimento 1. El grupo experimental se entrenó con el programa dual n-back los días hábiles, con una frecuencia de 2 sesiones diarias, y una duración aproximada de 1 hora por día. En total, el grupo experimental realizó 100 sesiones, con una duración aproximada de 50 horas, en 50 encuentros. Luego de 25 sesiones (Post test 1), de 50 sesiones (Post-test 2), de 75 sesiones (Post-test 3), y de 100 sesiones (Post-test 4), el grupo fue evaluado con los siguientes instrumentos en el siguiente orden: a) Test de Raven, Escala Avanzada; b) Test Amplitud de Dígitos; c) Test G; d) Prueba de Amplitud Lectora.

Por parte del grupo control, el mismo se entrenó las primeras 25 sesiones en la resolución de problemas aritméticos, como ya fue expuesto anteriormente, y luego sólo asistió para las mediciones de las 25, 50, 75 y 100 sesiones, las cuales se realizaron los mismos días que en el grupo experimental. Es necesario destacar que la asistencia para la toma de los instrumentos por parte del grupo experimental y del grupo control se efectuó en horarios diferentes, para evitar el contacto entre los dos grupos.

\section{Análisis de datos}

Se analizaron los datos con el mismo software, el mismo nivel de significación, y los mismos procedimientos con respecto a la determinación de pruebas paramétricas o no paramétricas. 
Panza Lombardo I Psicodebate 16 (1) | 49-82

\section{Resultados}

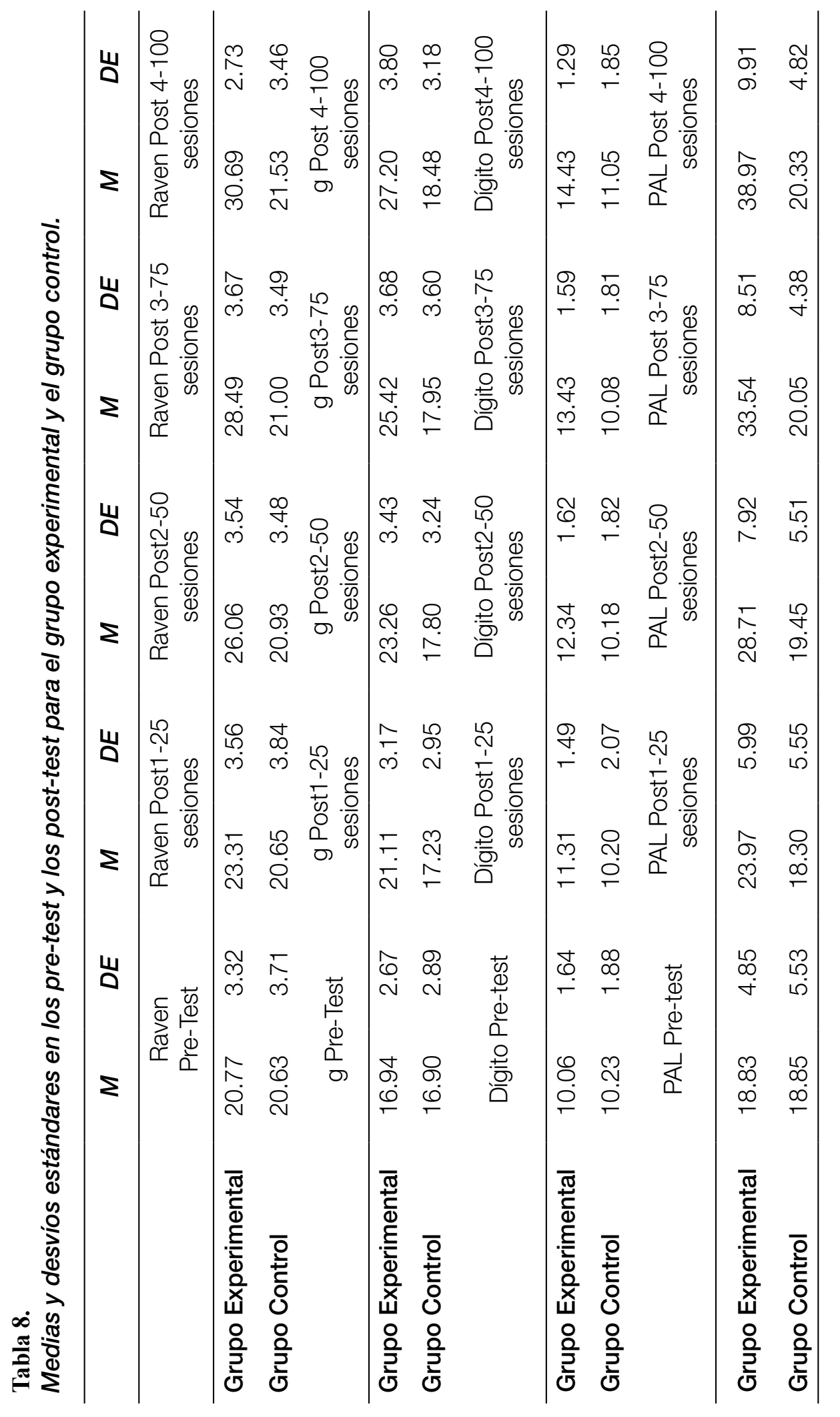


Con respecto a la inteligencia fluida se observa lo siguiente: en el test de Raven se apreciaron diferencias estadísticamente significativas en todas las comparaciones $(p<.05)$, con tamaños del efecto de magnitud media en la primera comparación, y elevada en las restantes. En el Test g se observaron diferencias estadísticamente significativas en todas las comparaciones $(p<.05)$, con un tamaño del efecto elevado.

En cuanto a la memoria de trabajo verbal, en el Test Dígito existieron aumentos significativos en todas las comparaciones post-test, con tamaños del efecto elevados para las primeras tres comparaciones, y medio para la última comparación. Mientras que en la Prueba de Amplitud Lectora se observaron diferencias estadísticamente significativas en todas las comparaciones, con tamaños del efecto medios en la primera comparación, y grandes en las restantes (Rosenthal, 1991).

Tabla 9.

Diferencias intra grupo en los post-test para el grupo experimental y el grupo control.

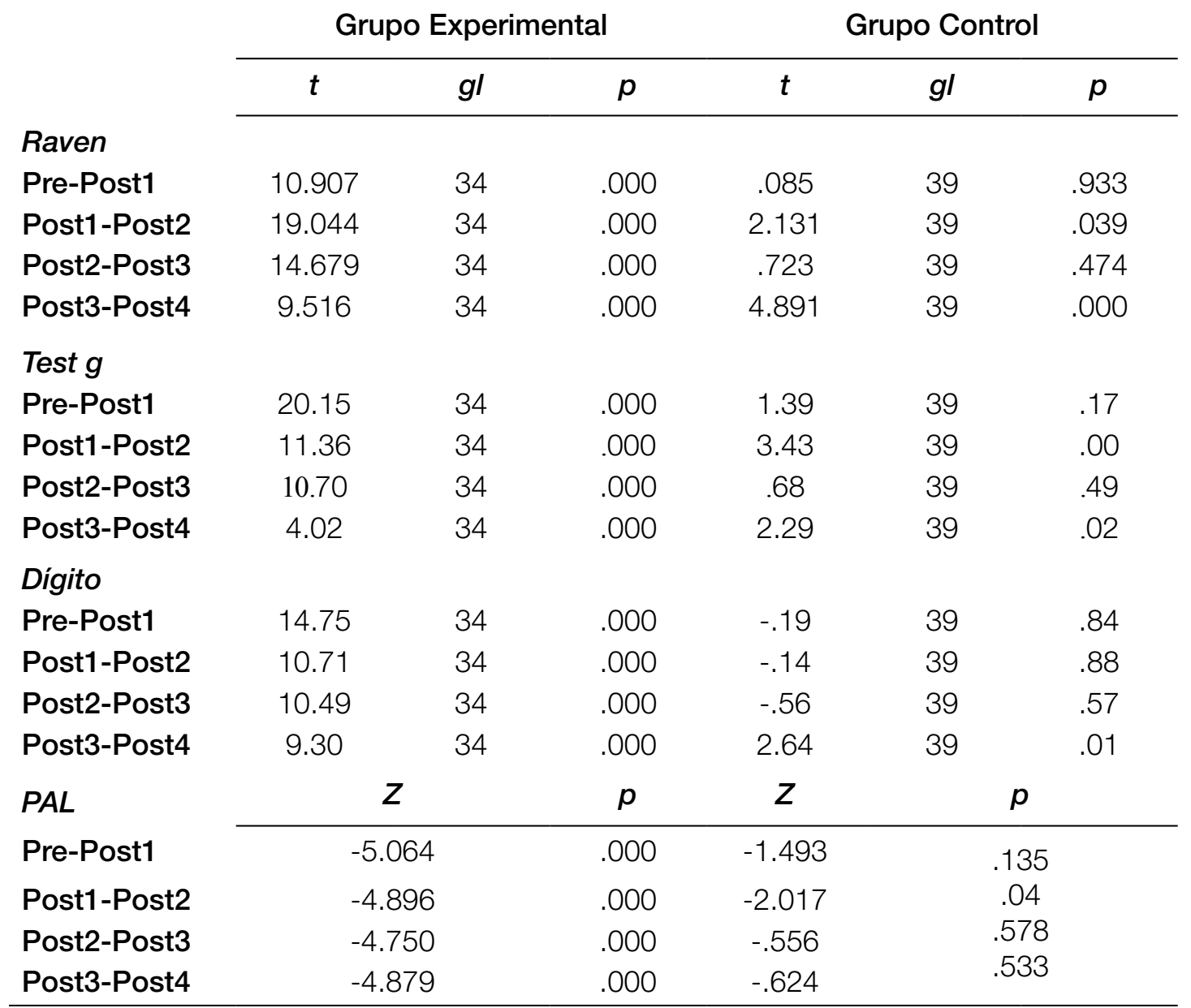


Panza Lombardo I Psicodebate 16 (1) | 49-82

Tabla 10.

Diferencia extra-grupo en los post-test con respecto a todas las variables.

\begin{tabular}{|c|c|c|c|c|c|}
\hline & $t$ & & $g l$ & $p$ & $d$ de Cohen \\
\hline \multicolumn{6}{|l|}{ Raven } \\
\hline Post test 1-25 & 3.100 & & 73 & .003 & .70 \\
\hline Post test 2-50 & 6.313 & & 73 & .000 & 1.44 \\
\hline Post test3-75 & 9.038 & & 73 & .000 & 2.06 \\
\hline Post test4-100 & 12.589 & & 73 & .000 & 2.87 \\
\hline \multicolumn{6}{|l|}{ Test $g$} \\
\hline Post test 1-25 & 5.487 & & 73 & .000 & 1.25 \\
\hline Post test 2-50 & 7.073 & & 73 & .000 & 1.61 \\
\hline Post test3-75 & 8.871 & & 73 & .000 & 2.02 \\
\hline Post test4-100 & 10.811 & & 73 & .000 & 2.46 \\
\hline \multicolumn{6}{|l|}{ Dígito } \\
\hline Post test 1-25 & 8.158 & & 73 & .000 & 1.86 \\
\hline Post test 2-50 & 5.401 & & 60.80 & .000 & 1.18 \\
\hline Post test3-75 & 5.751 & & 61.67 & .000 & 1.26 \\
\hline Post test4-100 & 3.112 & & 67.49 & .003 & .69 \\
\hline$P A L$ & $U$ & $w$ & $Z$ & $p$ & $r^{*}$ \\
\hline Post test 1-25 & 328 & 1148 & -4.006 & .000 & .46 \\
\hline Post test 2-50 & 207.5 & 1027.5 & -5.295 & .000 & .61 \\
\hline Post test3-75 & 61 & 881 & -6.848 & .000 & .79 \\
\hline Post test4-100 & 22 & 842 & -7.258 & .000 & .83 \\
\hline
\end{tabular}




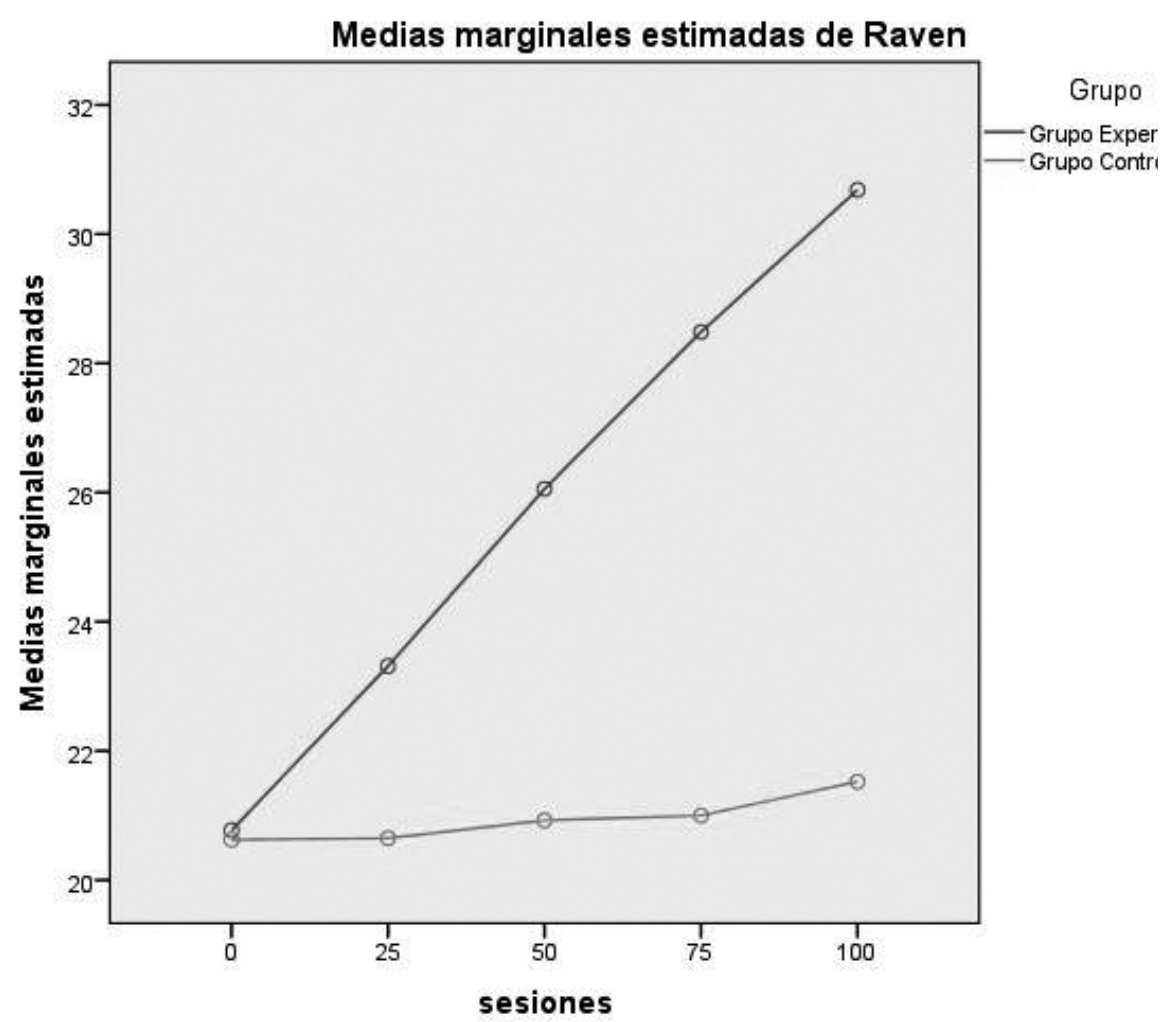

Figura 6. Medias del grupo experimental y control en el pre y en los post-test, para el test de Raven.

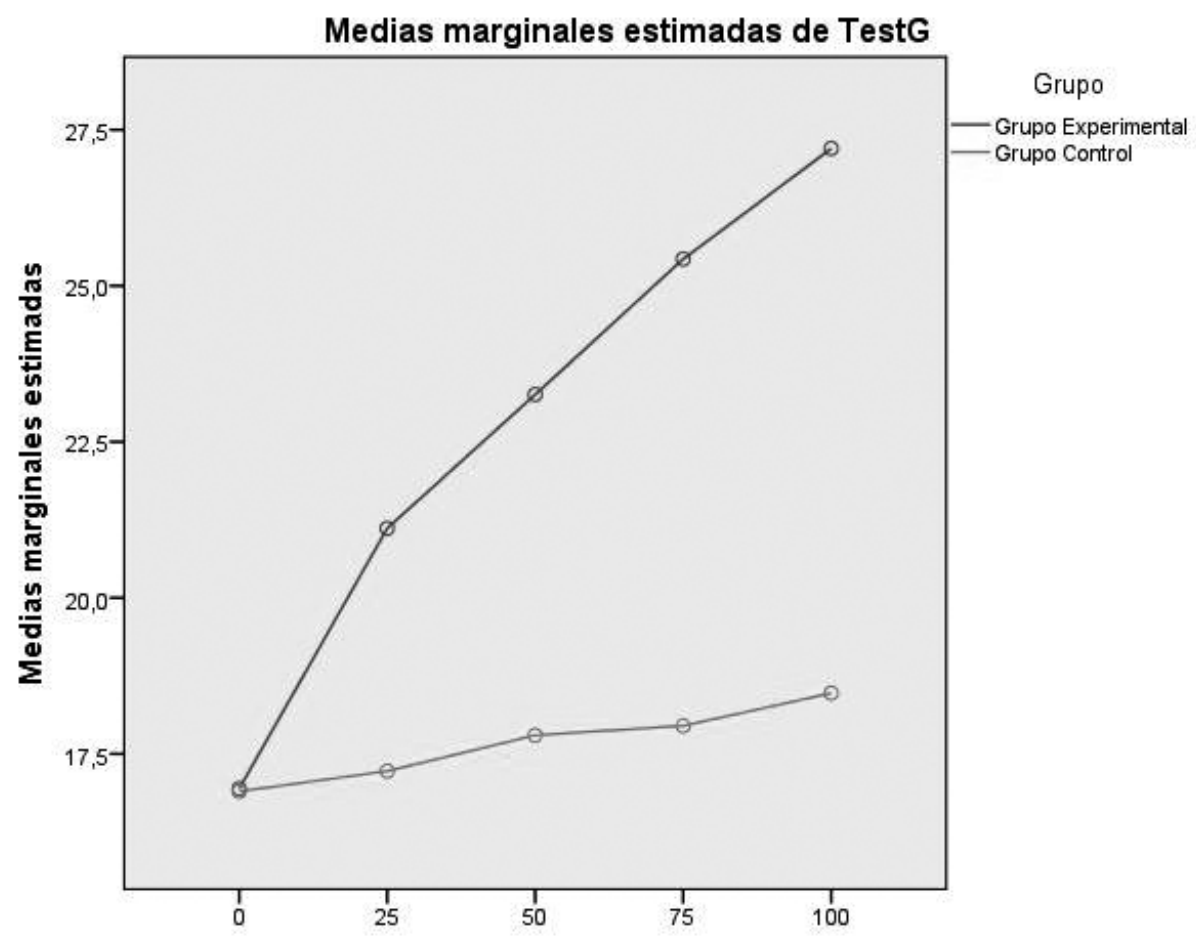

Figura 7. Medias del grupo experimental y control en el pre y los post tests, para el test $g$. 


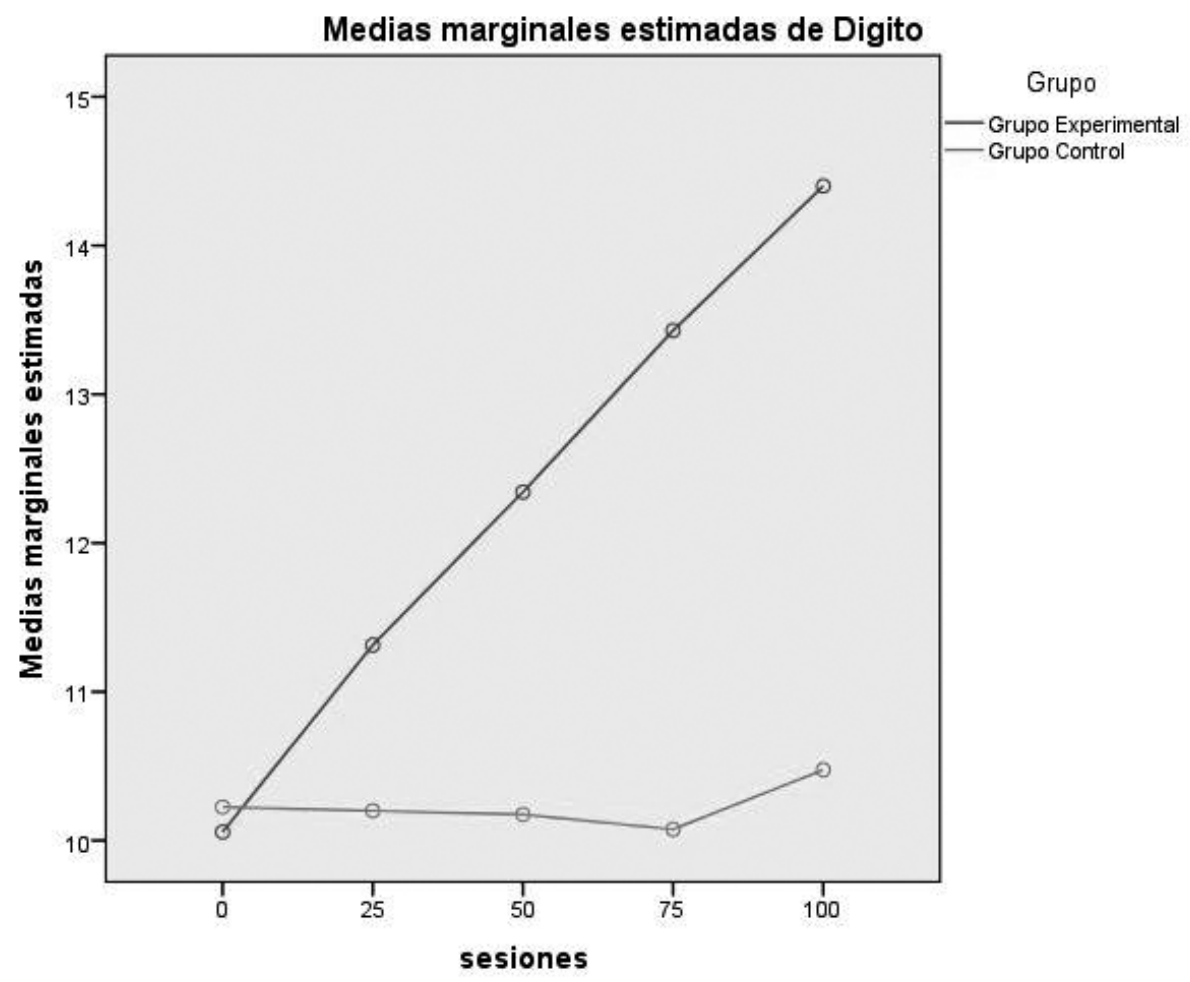

Figura 8. Medias del grupo experimental y control en el pre y los post tests, para el test de Amplitud de Dígitos.

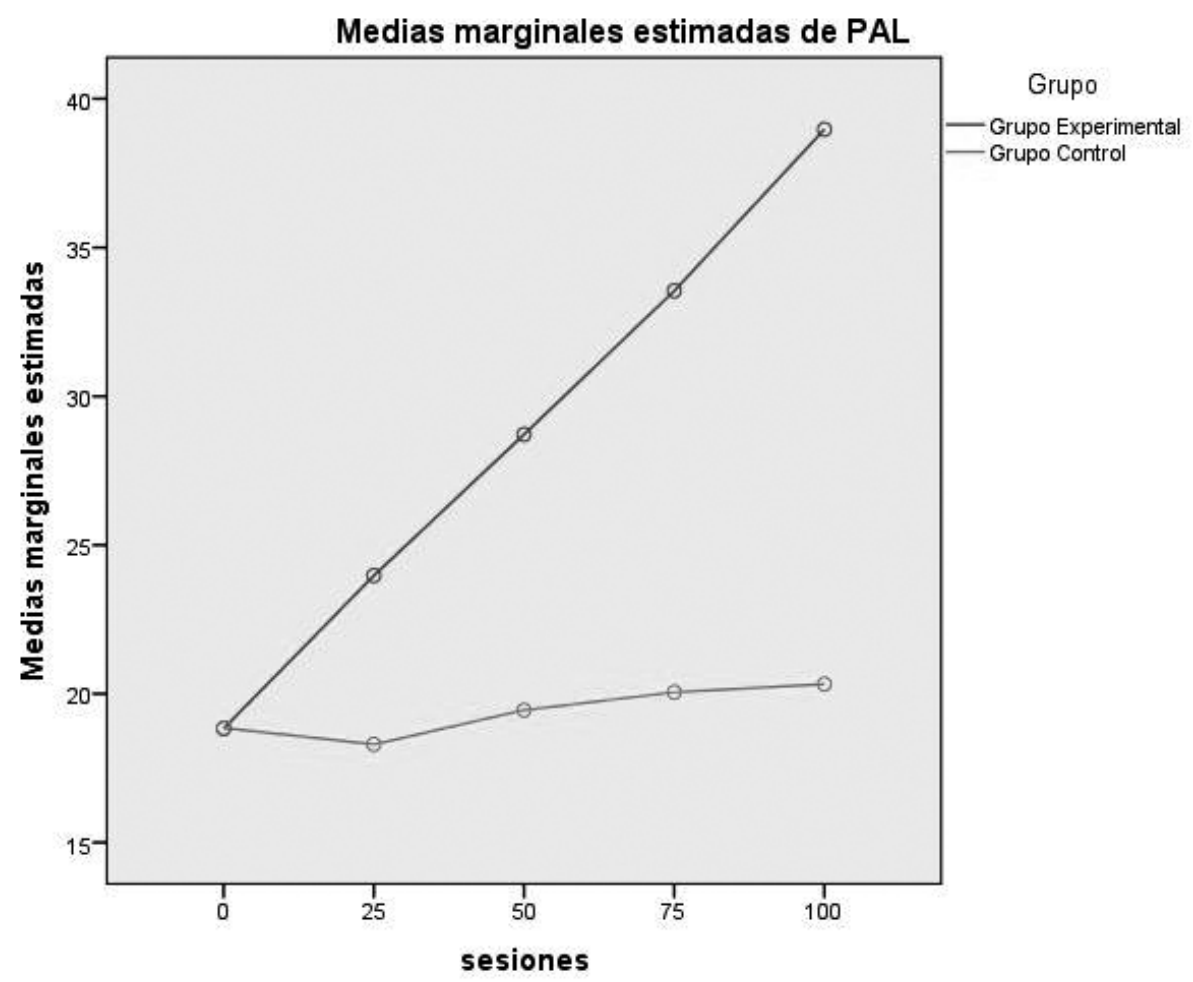

Figura 9. Medias del grupo experimental y control en el pre y los post tests, para la Prueba de Amplitud Lectora. 
En síntesis, los datos apoyan las hipótesis 3 y 4: Se registraron aumentos de la inteligencia fluida y de la memoria de trabajo mayores en el entrenamiento de 100 sesiones en comparación con el de 75 sesiones, en el entrenamiento de 75 sesiones en comparación con el de 50 sesiones, y en el entrenamiento de 50 sesiones en comparación con el de 25 sesiones, y en el entrenamiento de 25 sesiones.

\section{Estudio 3}

Con el experimento 3 se evaluaron las hipótesis 5 y 6 , las cuales afirmaban que existirían diferencias a favor del grupo experimental luego de 1, 2, 4 y 6 meses de culminado el entrenamiento, tanto en inteligencia fluida como en memoria de trabajo verbal.

\section{Participantes}

Se emplearon los mismos participantes de los experimentos 1, 2 y 3 .

\section{Instrumentos}

Se utilizaron los siguientes instrumentos en este orden de evaluación: a) Test de Raven, Escala Avanzada; b) Test Amplitud de Dígitos; c) Test G; d) Prueba de Amplitud Lectora.

\section{Diseño}

Diseño experimental, de series cronológicas múltiples, con grupo control.

\section{Procedimientos}

Al mes de terminado el entrenamiento del grupo experimental, se citó a todos los participantes para tomarle los cuatro instrumentos, en el mismo orden en el que fueron tomados anteriormente, realizándose lo mismo a los 2, 4, y 6 meses. Se presentaron dificultades de horarios y de fechas. Sin embargo, se pudo suplir las mismas y lograr que cada participante fuera medido en los mismos intervalos de tiempo.

\section{Análisis de datos}

Se utilizó el mismo software, el mismo nivel de significación y el mismo procedimiento para determinar la utilización de pruebas paramétricas o no paramétricas que en los estudios anteriores. 
Panza Lombardo I Psicodebate 16 (1) | 49-82

\section{Resultados}

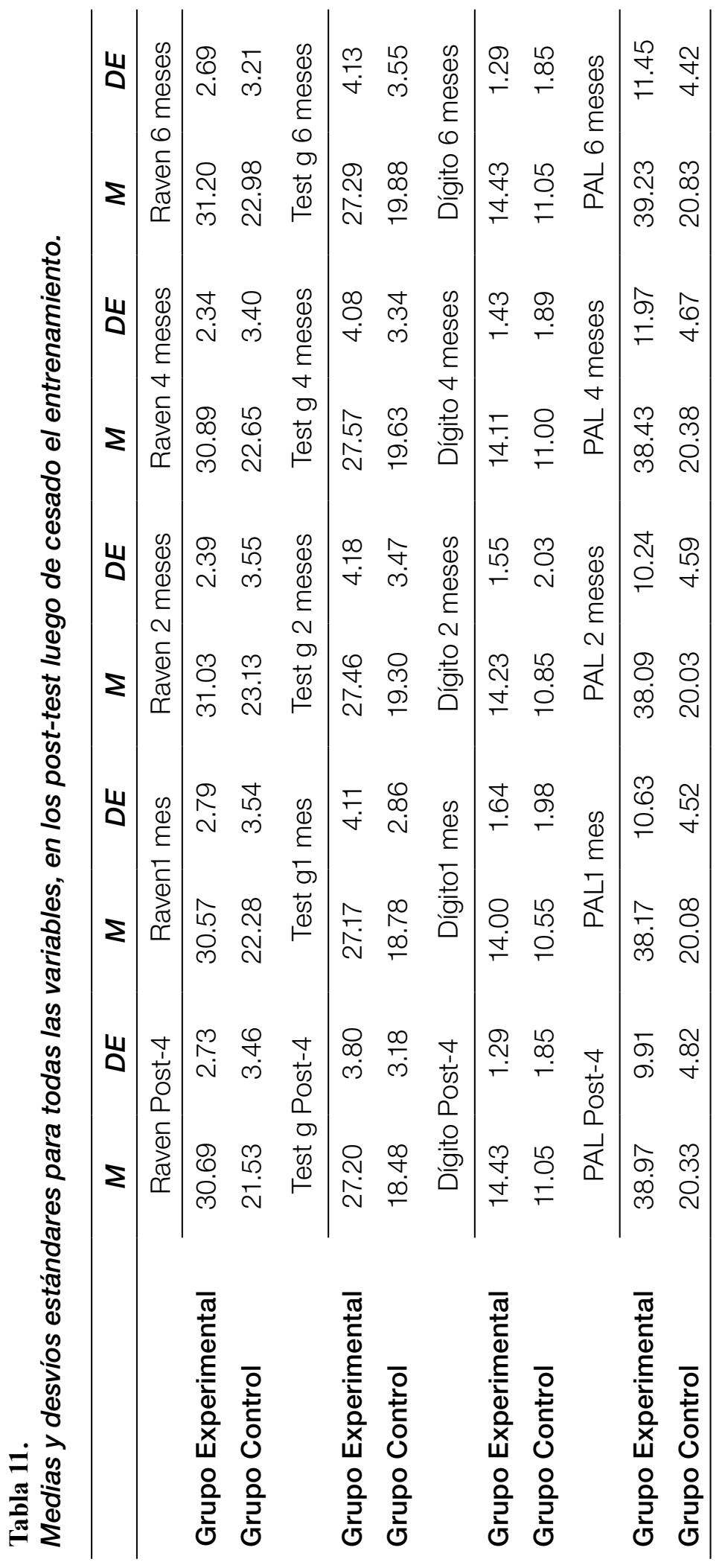


Tabla 12.

Comparaciones extra-grupo en todos los post-test luego de finalizado en entrenamiento.

\begin{tabular}{|c|c|c|c|c|c|}
\hline \multirow[b]{2}{*}{ Raven } & \multicolumn{2}{|l|}{$t$} & $g l$ & \multirow[t]{2}{*}{$p$} & \multirow[t]{2}{*}{$d$ de Cohen } \\
\hline & & & & & \\
\hline 1 mes & 11.149 & & 73 & .000 & 2.546 \\
\hline 2 meses & 11.414 & & 68.749 & .000 & 2.542 \\
\hline 4 meses & 12.321 & & 69.437 & .000 & 2.748 \\
\hline 6 meses & 11.904 & & 73 & .000 & 2.719 \\
\hline \multicolumn{6}{|l|}{ Test $g$} \\
\hline 1 mes & 10.116 & & 59.68 & .000 & 2.364 \\
\hline 2 meses & 9.227 & & 73 & .000 & 2.107 \\
\hline 4 meses & 9.249 & & 73 & .000 & 2.112 \\
\hline 6 meses & 8.349 & & 73 & .000 & 1.906 \\
\hline \multicolumn{6}{|l|}{ Dígito } \\
\hline 1 mes & 8.121 & & 73 & .000 & 1.855 \\
\hline 2 meses & 7.996 & & 73 & .000 & 1.826 \\
\hline 4 meses & 7.942 & & 73 & .000 & 1.814 \\
\hline 6 meses & 9.037 & & 73 & .000 & 2.064 \\
\hline$P A L$ & $U$ & $w$ & $z$ & $p$ & $r^{*}$ \\
\hline 1 mes & 42.5 & 862.5 & -7.008 & .000 & .80 \\
\hline 2 meses & 56 & 876 & -6.861 & .000 & .79 \\
\hline 4 meses & 69 & 889 & -6.728 & .000 & .77 \\
\hline 6 meses & 42.5 & 862.5 & -7.018 & .000 & .81 \\
\hline
\end{tabular}

Como puede apreciarse en la tabla 12, luego del paso de 1, 2, 4, y 6 meses se encontraron diferencias extra-grupo significativas tanto para la inteligencia fluida como para la memoria de trabajo verbal, manteniéndose un tamaño del efecto elevado. 


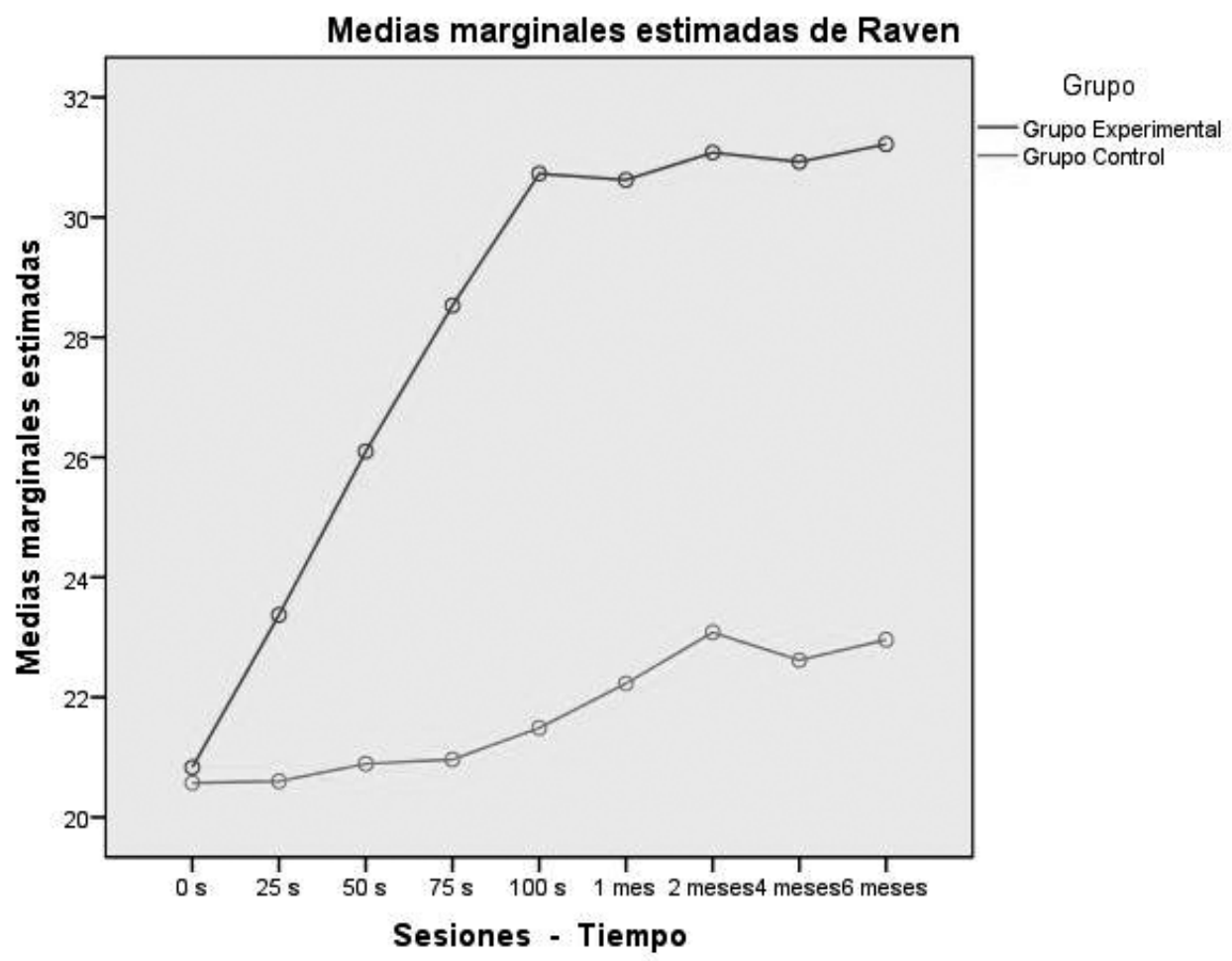

Figura 10. : Medias del grupo experimental y grupo control en todos los post-tests con respecto al test de Raven.

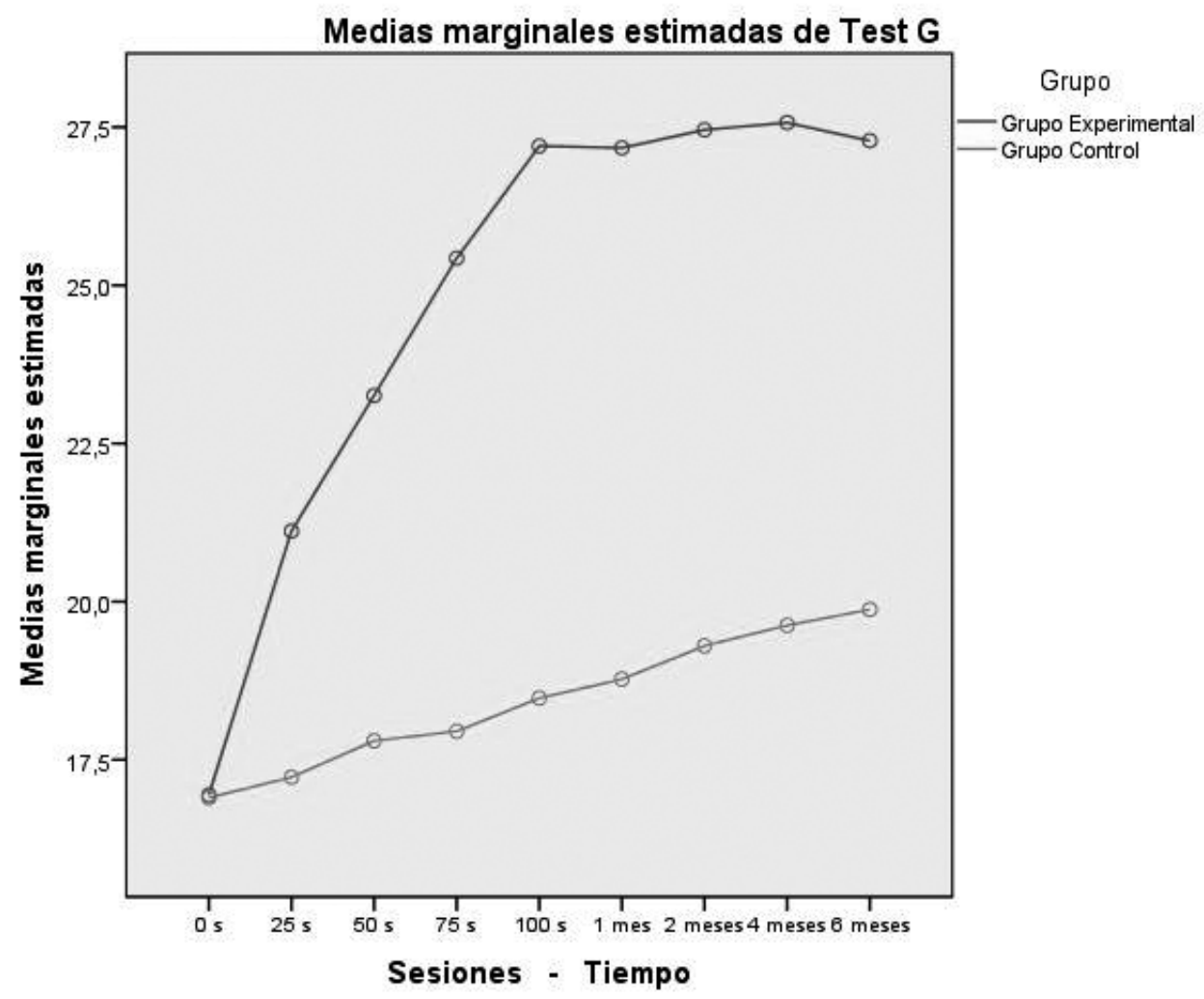

Figura 11. Medias en todos los post-tests del grupo experimental y control con respecto al test $\mathrm{g}$. 
En síntesis, los resultados son coherentes con las hipótesis 5 y 6, las cuales sostenían que existirían niveles de inteligencia fluida y de memoria de trabajo mayores en el grupo experimental que en el grupo control en todos los post-test efectuados luego de la finalización del entrenamiento.

\section{Discusión}

Jaeggi et al. (2008), utilizando el programa de entrenamiento dual n-back, reportaron aumentos en la inteligencia fluida y en la memoria de trabajo verbal. Estos resultados fueron cuestionados por Shipstead et al. (2010), debido a la ausencia de grupo control activo. Los autores sostuvieron que esta falta reducía la validez interna, dado que los resultados podían explicarse por efecto Hawthorne y/o por respuesta a la demanda.

A partir de lo antecedente, en el experimento 1 se pusieron a prueba las hipótesis 1 y 2 , las cuales afirmaban que el grupo experimental obtendría un aumento mayor en los niveles de inteligencia fluida y en los niveles de memoria de trabajo verbal que el grupo control activo. Las dos hipótesis fueron confirmadas por los resultados, obteniéndose mayores aumentos en el grupo experimental que en el grupo control, tanto para los niveles de inteligencia fluida como para los niveles de memoria de trabajo verbal. Los resultados apoyan la postura de Jaeggi et al. (2008), con respecto a la posibilidad de lograr una transferencia del entrenamiento dual n-back en la inteligencia fluida y en la memoria de trabajo verbal. Consiguientemente, contradicen las afirmaciones de Shipstead et al. (2010), acerca de que dichos resultados puedan deberse al efecto Hawthorne y/o a la respuesta a la demanda. También es preciso destacar que, a diferencia de los datos obtenidos por Jaeggi et al. (2008), se obtuvieron ganancias en la Prueba de Amplitud Lectora.

Los resultados apoyan la afirmación de que el programa genera aumentos en la inteligencia fluida y en la memoria de trabajo verbal, acordes con los metaanálisis de Au et al. (2014), Karbach y Verhaeghen (2014) y Schwaighofer, Fischer y Bühner (2015), mientras que sugieren que, a pesar de la utilización del grupo control, se obtienen efectos significativos y positivos.

El segundo experimento tuvo como finalidad evaluar los efectos de 25, 50, $75 \mathrm{y}$ 100 sesiones de entrenamiento en el programa dual n-back, teniendo en cuenta los datos contradictorios con respecto al efecto por bloque de sesión de entrenamiento (Jaeggi et al., 2008; Jaeggi et al., 2011). Tanto para la inteligencia fluida como para la memoria de trabajo verbal se obtuvieron resultados positivos, aceptándose las hipótesis 3 y 4 . Los resultados sugieren que el efecto del entrenamiento es sumativo, por lo que hace aún más plausible la posibilidad de aumentar la inteligencia fluida y la memoria de trabajo por medio de entrenamiento. Por 
lo cual este resultado se vuelve de interés práctico en cuanto a la cantidad de aumento que puede lograrse por medio de entrenamiento, si tenemos en cuenta los elevados tamaños del efecto obtenidos.

En el tercer experimento, por el cual se evaluaron las hipótesis 5 y 6 , se obtuvieron diferencias estadísticamente significativas a favor del grupo control en todas las medidas de inteligencia fluida y de memoria de trabajo. No existían datos con respecto a la permanencia del efecto de la tarea de entrenamiento dual $n$-back. Sin embargo, otros programas de entrenamiento habían obtenido la permanencia de los efectos en la inteligencia fluida desde 3 meses hasta 2 años (Ball et al., 2002; Borella et al., 2010; Jaeggi et al., 2011; Klauer, Willmes \& Phye, 2002; Plemons, Willis \& Baltes, 1978), y permanencias en la memoria de trabajo verbal de 6 a 8 meses, las cuales se perdían luego de un año de cesado el entrenamiento (Buschkuehl et al., 2008). Por lo tanto, los resultados apoyan la posibilidad de que las ganancias en inteligencia fluida y en memoria de trabajo se mantengan.

Existen limitaciones metodológicas que deben enumerarse. En primer lugar se podría cuestionar el tipo de grupo control activo que fue empleado en el experimento 1. Se debe tener en cuenta que mientras los integrantes del grupo experimental se entrenaron por medio de un software, el cual automáticamente les informaba acerca de sus rendimientos, los integrantes del grupo control activo se entrenaron mediante material impreso, el cual era de simple corrección, pero que evidentemente no contaba con la rapidez y comodidad del software. Por lo tanto, es posible que el programa dual n-back haya producido un efecto positivo en los participantes experimentales mayor al del programa de entrenamiento impreso. Si tal hecho fuese cierto, no podría descartarse el efecto Hawthorne. Esto debe tenerse en cuenta, dado que es probable que actualmente, para la mayoría de las personas, máxime para adultos jóvenes, sea más motivador estar frente a una computadora que frente a material impreso.

Otro aspecto criticable es que el grupo control activo no se mantuvo durante las 100 sesiones. Esto era deseable, principalmente dados ciertos resultados (Dougherty et al., 2015), pero temporalmente poco factible y hubiera podido hacer peligrar la factibilidad de toda la investigación. Podría aducirse que la ausencia de contacto con el grupo control luego de las 25 sesiones produjo en sus miembros una desmotivación, la cual disminuyó el esfuerzo empleado en responder a los instrumentos.

Un aspecto que claramente limita el alcance de la investigación es su validez externa. Los participantes no fueron elegidos probabilísticamente y, por otra parte, poseen una homogeneidad grande, por lo cual la generalización de los resultados obtenidos es restringida.

Sin embargo, la crítica más fuerte que puede realizarse a esta investigación no radica en si se produjo o no un aumento en las medidas causado por el programa 
de entrenamiento, sino en qué ha aumentado. Si bien no puede argüirse efecto testretest, puesto que se utilizaron grupos control para evitarlos, sí podría decirse que la tarea $d$ ual $n$-back generó una mayor facilidad para responder a los instrumentos de papel y lápiz, de computadora y de dictado, sin ninguna aplicación práctica ulterior. Quizás, la mejora no esté en el mecanismo de la inteligencia fluida o en el de la memoria de trabajo verbal, sino en la aptitud para enfrentarse con pruebas. Es posible que el hecho de que la tarea dual n-back sea de índole ininterrumpida generara un efecto de habituación ante tales pruebas, y que por esto se expliquen los niveles mayores en los instrumentos de medición. Tal vez no exista una diferencia tan grande entre la tarea dual n-back y los instrumentos evaluados, y quizás lo que haya mejorado haya sido la capacidad para resolver los instrumentos y no los mecanismos neurocognitivos de la inteligencia fluida y de la memoria de trabajo. Existen numerosos autores que apoyan la hipótesis de una disociación entre las medidas de inteligencia y el mecanismo inteligencia (Bors \& Vigneau, 2003; Jensen, 1969, 1998; Mody, 2009; Rushton \& Jensen, 2010; Shipstead et al., 2010; te Nijenhuis et al., 2007). Al respecto, la investigación de Colom et al., (2013) reportaron que los aumentos en los test de inteligencia fluida realmente se deben a aumentos en los procesos visuoespaciales y no a aumentos en cuanto al constructo inteligencia. Sería interesante poder investigar más con respecto a esta perspectiva, máxime teniendo en cuenta que también se han producido aumentos en la memoria de trabajo verbal, aspecto no ligado con procesos visuoespaciales.

La última crítica señala el camino que las investigaciones podrían seguir en la temática. Sería muy útil que se efectuaran investigaciones similares a la presente, pero que utilizaran, además de los instrumentos psicométricos, medidas con neuroimágenes antes, durante y después de la intervención. Una investigación ideal implicaría poder combinar varias medidas neurológicas de la inteligencia fluida y de la memoria de trabajo verbal, como pueden ser la Resonancia Magnética Nuclear (fRMI) para notar cambios en la activación de las zonas corticales, la Tomografía de Emisión de Positrones (PET), para determinar cambios en la activación y modificaciones en el metabolismo de la glucosa, la Tractografía por Resonancia Magnética, para comparar la eficiencia de las conexiones neuronales, y el Electroencefalograma (EEG), para comparar la eficiencia neurológica. Sería muy interesante y pertinente efectuar con estas medidas un diseño pre-test posttest con grupo de control activo.

Por otra parte, una alternativa menos costosa es la de efectuar el entrenamiento en una población que esté sometida a numerosos e iguales exámenes académicos, como pueden ser los integrantes de alguna institución educativa, y comparar los rendimientos académicos de ambos grupos luego del programa de entrenamiento, utilizando un grupo control activo con otra tarea informática. 


\section{Referencias}

Ackerman, P. L., Beier, M. E. \& Boyle, M. O. (2005). Working memory and intelligence: The same or different constructs? Psychological Bulletin, 131(1), $30-60$.

Alloway, T. P. (2009). Working memory, but no IQ, predicts subsecuent learning in children with learning difficulties. Psychological Assessment, 25(2), 92-98.

Alloway, T. P. \& Alloway, R. G. (2010). Investigating the predictive roles of working memory and IQ in academic attainment. Journal of Experimental Child Psychology, 106, 20-29.

Au, J., Buschkuehl, M. , Duncan, G. J. \& Jaeggi, S. M. (2015). There is no convincing evidence that working memory training is NOT effective: A reply to Melby-Lervåg and Hulme (2015). Psychonomic Bulletin \& Review, 23(1), 331-337.

Au, J. Sheehan, E., Tsai, N. , Duncan, G. J. , Buschkuehl, M. \& Jaeggi, S. M. (2014). Improving fluid intelligence with training on working memory: a metaanalysis. Psychonomic Bulletin and Review, 22(2), 366-377.

Ball, K. , Berch, D. B., Helmers, K. F., Jobe, J. B., Leveck, M. D., Marsiske, M., ... Willis, S. L. (2002). Effects of Cognitive Training Interventions With Older Adults. Journal of the American Medical Association, 288(18), 2271-2281.

Barbey, A. K., Colom, R., Paul, E. J., \& Grafman, J. (2014). Architecture of fluid intelligence and working memory revealed by lesion mapping. Brain Structure and Function, 219(2), 485-494.

Barreyro, J. P., Burin, D. I. \& Duarte, D. A. (2009). Capacidad de la memoria de trabajo verbal. Validez y fiabilidad de una tarea de amplitud lectora. Interdisciplinaria, 26(2), 207-228.

Basak, C., Boot, W. R., Voss, M. W. \& Kramer, A. F. (2008). Can training in a real-time strategy video game attenuate cognitive decline in older adults? Psychology and Aging, 23(4), 765-777.

Borella, E., Carretti, B., Riboldi, F. \& De Beni, R. (2010). Working memory training in older adults: evidence of transfer and maintenance effects. Psychology and Aging, 25(4), 767-778. 
Bors, D. A., \& Vigneau, F. (2003). The effects of practice on Raven's Advanced Progressive Matrices. Learning and Individual Differences, 13(4), 1-22.

Buschkuehl, M., Jaeggi, S. M., Kobel, A. \& Perrig, W. J. (2007). BrainTwister - A collection of cognitive training tasks. Berna: Universität Bern.

Burgess, G. C., Gray, J. R., Conway, A. R. A. \& Braver, T. S. (2011). Neural mechanism of interference control underlie the relashionship between fluid intelligence and working memory span. Journal of Experimental Psychology, General, 140(4), 674-692.

Cattell, R. B. (1987). Intelligence: Its structure, growth, and action. Nueva York: Elsevier Science Pub. Co.

Cattell, R. B. \& Cattell, A. K. S. (1973/2001). Manual test Factor “g”. Escalas 2 y 3. Madrid: Tea Ediciones.

Carroll, J. B. (1993). Human cognitive abilities: A survey of factor-analytic studies. Nueva York: Cambridge University Press.

Colom, R., Privado, J., García, L. F., Estrada, E., Cuevas, L. \& Shih, P. C. (2015). Fluid intelligence and working memory capacity: Is the time for working on intelligence problems relevant for explaining their large relationship? Personality and Individual Differences, 79, 75-80.

Colom, R., Román, F. J., Abad, F. J., Shih, P. C., Privado, J., ... Jaeggi, S. M. (2013). Adaptative n-back training does not improve fluid intelligence at construct level: Gains on individual tests suggest that training may enhance visuospacial processing. Intelligence, 41, 712-727.

Cowles, M. P. (1974). $\mathrm{N}=35$ : A rule of thumb for psychological researchers. Perceptual and Motor Skills, 38, 1135-1138.

Darst, B. F., Koscic, R. L., Hermann, B. P., La Rue, A., Sager, M. A., Johnson, S. C. \& Engelman, C. D. (2015). Heritability of cognitive traits among sibilings with a parental history of Alzheimer's disease. Journal of Alzheimers Disease, 45(4), 1149-1155.

Davies, G., Tenesa, A., Payton, A., Yang, J., Harris, S. E., Liewald, D. ... Deary, I. J. (2011). Genome-wide association studies establish that human intelligence is highly heritable and polygenic. Molecular Psychatry, 16(10), 996-1005. 
Deary, I. J., Strand, S., Smith, P. \& Fernandes, C. (2007). Intelligence and educational achievement. Intelligence, 35(1), 13-21.

Dougherty, M. R., Hamovitz, T. \& Tidwell, J. W. (2015). Reevaluating the effectiveness of n-back training on transfer throught the Bayesian lens: Support for the null. Psychonomic bulletin and review, 23(1), 1-11

Elosúa, M. R., Gutierrez, F., García Madruga, J. A., Luque, J. L. \& Gárate, M. (1996). Adaptación española del —reading span task de Daneman y Carpenter. Psicothema, 8(2), 383-395.

Engel de Abreu, P. M. J., Conway, A. R. A. \& Gathercole, S. E. (2010). Working memory and fluid intelligence in young children. Intelligence, 38, 552-561.

Engle, R. W., Tuholski, S. W., Laughlin, J. E. \& Conway, A. R. A. (1999). Working memory, short-term memory, and general fluid intelligence: A latent-variable approach. Journal of Experimental Psychology: General, 128(3), 309-331.

Eysenck, H. J. \& Eysenck, S. B. G. (1985/1997). Cuestionario revisado de personalidad de Eysenck. Versiones completa(EPQ-R) y abreviada(EPQ-RS). Madrid: Tea Ediciones.

Gignac, G. E. \& Watkins, M. W. (2015). There may be nothing special about the association between working memory capacity and fluid intelligence. Intelligence, 52, 18-23.

Gray, J. R., Chabris, C. F. \& Braver, T. S. (2003). Neural mechanism of general fluid intelligence. Nature Neuroscience, 6(3), 316-322.

Horn, J. L. (1972). State, trait and change in dimensions of intelligence. British Journal of Educational Psychology, 42, 159-186.

Jaeggi, S. M., Buschkuehl, M., Jonides, J. \& Perrig, W. J. (2008). Improving fluid intelligence with training on working memory. Proceedings of the National Academy of Sciences, 105, 6829-6833. doi:10.1073/pnas.0801268105

Jaeggi, S. M., Buschkuehl, M., Jonides, J. \& Shah, P. (2011). Short- and long-term benefits of cognitive training. Proceedings of National Academy of Sciences, 10(1073), 1181-1186.

Jaeggi, S. M., Studer-Luethi, B., Buschkuehl, M., Su, Y., Jonides, J. \& Perrig, W. J. (2010). The relationship between n-back performance and matrix reasoning -- implications for training and transfer. Intelligence, 38(6), 625-635. 
Jensen, A. R. (1969). How much can we boost I.Q. and scholastic achievement? Harvard Educational Review, 33, 1-12.

Jensen, A. R. (1998). The g factor: The science of mental ability. Westport, CT: Praeger.

Julius, E. H. (1994). Matemáticas Rápidas. Bogotá: Norma.

Kane, M. J. \& Engle, R. W. (2002). The role of prefrontal cortex in workingmemory capacity, executive attention, and general fluid intelligence: An individual-differences perspective. Psychonomic Bulletin \& Review, 9(4), 637-671.

Kane, M. J., Conway, A. R. A. \& Hambrick, D. Z. (2005). Working memory capacity and fluid intelligence are strongly related constructs: comments on Ackerman, Beier and Boyle(2005). Psychological Bulletin, 131(1), 66-71.

Karbach, J. \& Verhaeghen, P. (2014). Making working memory work. A metaanalysis of executive-control and working memory training in older adults. Psychological Science, 25(11), 2027-2037.

Kaufman, A. S., Kaufman-Packer, J. L., Mc Lean, J. E. \& Reinolds, C. R. (1991). Is the pattern of intellectual growth and decline across the adult life span different for men and women? Journal of Clinical Psychology, 47, 802-812.

Klauer, K. J., Willmes, K. \& Phye, G. D. (2002). Inducing Inductive Reasoning: Does it transfer to Fluid Intelligence? Contemporary Educational Psychology, 27, 1-25.

Klingberg T., Forssberg H. \& Westerberg, H. (2002). Training of working memory in children with ADHD. Journal of Clinical Experimental Neuropsycholy, 24(6), 781-791.

Loosli, S. V., Buschkuehl, M., Perrig, W. J. \& Jaeggi, S. M. (2012). Working memory training improves reading processes in typically developing children. Child Neuropsychology, 18(1), 62-78.

Melby-Lervåg, M. \& Hulme, C. (2013). Is working memory training effective? A meta-analytic review. Developmental Psychology, 49(2), 270-291.

Melby-Lervåg, M. \& Hulme, C. (2015). There is no convincing evidence that working memory training is effective: A reply to Au et al. (2014) and Karbach and Verhaeghen (2014). Psychonomic Bulletin and Review, 23(1), 324-330. 
Mody, D. E. (2009). Can intelligence be increased by training on a task of working memory? Intelligence, 37(4), 327-328.

Oberauer, K., Suß, H. M., Schulze, R., Wilhelm, O. \& Wittmann, W. W. (2000). Working memory capacity - facets of a cognitive ability construct. Personality and Individual Differences, 29, 1017-1045.

Plemons, J. K., Willis, S. L. \& Baltes, P. B. (1978). Modificability of Fluid Intelligence in Aging: A Short-Term Longitudinal Training Approach. Journal of Gerontology, 33 (2), 224-231.

Raven, J. C., Court, J. H. \& Raven, J. (1976/1999). Test de Matrices Progresivas. Escala Avanzada. Buenos Aires: Paidós.

Rojas-Barahona, C. A., Förster, C. E., Moreno-Ríos, S. \& McClelland, M. (2015). Improvement of working memory in preschoolers and its impact on early literacy skills: a study in deprived communities of rural and urban areas. Early Education Development, 26(5-6), 871-892.

Rosen, V. M. \& Engle, R. W. (1997). Forward and backward serial recall. Intelligence, 25(1), 37-47.

Rosenthal, R. (1991). Meta-analytic procedures for social research. Newbury Park(CA): Sage.

Rushton, J. P. \& Jensen, A. R. (2010). The rise and the fall of the Flynn Effect as a reason to expect narrowing of the Black-White IQ gap. Intelligence, 38, 213-219.

Salthouse, T. A. \& Babcock, R. L. (1991). Decomposing adult age differences in working memory. Developmental Psychology, 27(5), 763-776.

Schmidt, F. L. (2009). Select on intelligence. En E. A. Locke (Ed.), Handbook of principles of organizational behavior (pp. 3-18) West Sussex: Willey. Schmidt, F. L. \& Hunter, J. E. (1998). The validity and utility of selection methods in personnel psychology: Practical and theoretical implications of 85 years of research findings. Psychological Bulletin, 124(2), 262-274.

Schmidt, F. L. \& Hunter, J. E. (2004). General mental ability in the world of work: occupational attainment and job performance. Journal of Personality and Social Psychology, 86(1), 162-173.

Schwaighofer, M., Fischer, F. \& Bühner, M. (2015). Does working memory 
training transfer? A meta-analysis including training condition as moderators. Educational Psychologist, 50(2), 138-166.

Shipstead, Z., Redick, T. S. \& Engle, R. W. (2010). Does working memory training generalize? Psychologica Belgica, 50(3-4), 245-276.

te Nijenhuis, J., van Vianen, A. E. M. \& van der Flier, H. (2007). Score gains on g-loaded tests: No g. Intelligence, 35, 283-300.

Titz, C. \& Karbach, J. (2014). Working memory and executive functions: effects of training on academic achievement. Psychological Research, 78(6), 852-868.

Tranter, L. J. \& Koutstaal, W. (2008). Age and flexible thinking: an experimental demonstration of the beneficial effects of increased cognitively stimulating activity on fluid intelligence in healthy older adults. Neuropsycholgy, Development and Cogniton, Secction B, Aging, Neuropsychology and Cognition, 15(2), 184-207.

Unsworth, N. (2010). On the division of working memory and long-therm memory and their relation to intelligence: A latent variable approach. Acta psychologica, 134(1), 16-28.

van den Noort, M. , Haverkort, M. , Bosch, P. , \& Hugdahl, K. (2006). Is there a decline in verbal working memory over age? Europe's Journal of Psychology, 2(2).

Watkins M. W., Lei, P. \& Canivez G. L. (2007). Psychometric intelligence and achievement: A cross-lagged panel analysis. Intelligence, 35 (1), 5968-5968.

Wechsler, D. (1984). Test de Inteligencia para adultos. Buenos Aires: Paidós.

Wiley, J. \& Jaroz, A. F. (2012). How working memory capacity affects problem solving. En B. H. Ross (Ed.), Psychology of Learning and Motivation (vol. 56, pp. 185-228). San Diego, CA: Accademic Press.

Yuan, K., Steedle, J., Shavelson, R., Alonzo, A. \& Oppezzo, M. (2006). Working memory, fluid intelligence, and science learning. Educational Research Review, 1, 83-98. 
